\title{
Machine Fault Detection Based on Filter Bank Similarity Features Using Acoustic and Vibration Analysis
}

\author{
Mauricio Holguín-Londoño, ${ }^{1}$ Oscar Cardona-Morales, ${ }^{2}$ Edgar F. Sierra-Alonso, ${ }^{3}$ \\ Juan D. Mejia-Henao, ${ }^{4}$ Álvaro Orozco-Gutiérrez, ${ }^{1}$ and German Castellanos-Dominguez ${ }^{3}$ \\ ${ }^{1}$ Automatic Research Group, Universidad Tecnológica de Pereira, Pereira, Colombia \\ ${ }^{2}$ Technological and Environmental Advances Research Group, Universidad Católica de Manizales, Colombia \\ ${ }^{3}$ Signal Processing and Recognition Group, Universidad Nacional de Colombia, Manizales, Colombia \\ ${ }^{4}$ Universidad Nacional de Colombia, Manizales, Colombia
}

Correspondence should be addressed to Oscar Cardona-Morales; ocardonam@ucm.edu.co and German Castellanos-Dominguez; cgcastellanosd@unal.edu.co

Received 19 February 2016; Revised 10 May 2016; Accepted 8 June 2016

Academic Editor: Weihua Li

Copyright (c) 2016 Mauricio Holguín-Londoño et al. This is an open access article distributed under the Creative Commons Attribution License, which permits unrestricted use, distribution, and reproduction in any medium, provided the original work is properly cited.

\begin{abstract}
Vibration and acoustic analysis actively support the nondestructive and noninvasive fault diagnostics of rotating machines at early stages. Nonetheless, the acoustic signal is less used because of its vulnerability to external interferences, hindering an efficient and robust analysis for condition monitoring $(\mathrm{CM})$. This paper presents a novel methodology to characterize different failure signatures from rotating machines using either acoustic or vibration signals. Firstly, the signal is decomposed into several narrowband spectral components applying different filter bank methods such as empirical mode decomposition, wavelet packet transform, and Fourier-based filtering. Secondly, a feature set is built using a proposed similarity measure termed cumulative spectral density index and used to estimate the mutual statistical dependence between each bandwidth-limited component and the raw signal. Finally, a classification scheme is carried out to distinguish the different types of faults. The methodology is tested in two laboratory experiments, including turbine blade degradation and rolling element bearing faults. The robustness of our approach is validated contaminating the signal with several levels of additive white Gaussian noise, obtaining high-performance outcomes that make the usage of vibration, acoustic, and vibroacoustic measurements in different applications comparable. As a result, the proposed fault detection based on filter bank similarity features is a promising methodology to implement in CM of rotating machinery, even using measurements with low signal-to-noise ratio.
\end{abstract}

\section{Introduction}

Condition monitoring (CM) for rotating machinery is becoming an essential task that allows detecting faults at early stages, preventing unexpected damage and catastrophic accidents. In machine fault diagnoses, the vibration signal analysis is the most widely used nondestructive technique for extracting relevant information. Recently, data acquisition systems using acoustic signals have also gained demand over other noncontact measurement techniques when the sensor locations on the machine are unavailable or the measurement procedure has a high risk for workers [1]. Nonetheless, acoustic signals are more vulnerable to environmental noise than vibration responses [2], making employing signal preprocessing techniques necessary to reduce the undesirable interferences and improve the low signal-to-noise ratio (SNR) [3]. Furthermore, the vast majority of reported acousticbased $\mathrm{CM}$ are focused on visual inspections, eluding to incorporate the signal preprocessing into automatic diagnoses systems [4].

With the aim of enhancing the signal quality influenced by noisy environments, different preprocessing methods must be used. Due to the fact that wide class of machinery faults is defined by spectrally localized energies over narrow subbands, the filter bank methods (FBM) are employed as a common suitable preprocessing method for acoustic [1, 
5] and vibration signals [6]. Thus, CM usually includes a filter bank stage that splits the measured signal into a set of narrow spectral bands, which concentrate the information within a limited bandwidth related to the machine fault under consideration. In particular, wavelet packet transform (WPT) and empirical mode decomposition (EMD) are frequently applied as presented in $[2,7]$.

However, selection of the representative spectral components relies commonly on a priori knowledge available regarding the fault signatures, assuming each frequency band at which signatures may appear [8]. By instance, it is highly expected that bearing and gear faults appear at high frequencies. However, this knowledge is not accessible for most of the machine faults. Accordingly, there is a need for a measure that can identify the most representative spectral bands and hence perform a reliable assessment of the machine condition for a wide class of faults.

To date, several approaches have been developed to select the most discriminating narrow bands, which are mainly based on the use of similarity distances. In particular, the detection of impulsive behavior can be carried out using different measures of the relationship between narrow-band components. The structure of proposed correlation estimators can range from the baseline Pearson's correlation coefficient [9], merit index based on skewness [10], and spectral kurtosis [8] to more elaborate quantile-quantile plot-based selectors [11] and entropy-based indexes [12]. Nonetheless, the majority of these approaches rely on assumptions of stationarity for the correlated processes. This model may be not suitable for an extensive variety of nonstationary faults having impulsive or dynamics confined in the time domain.

In this paper, a similarity measure is introduced (termed cumulative spectral density index, CSDI) that deals with nonstationary estimates for the pairwise relationship measure between each narrow-band spectral component and the acquired raw signal, aiming to improve the detection of faults like unbalance, misalignment, and bearing faults. For the sake of comparison, we contrast the introduced CSDI with the baseline correlation index and cross-entropy value proposed for nonstationary analysis in [13]. As filter bank methods, we analyze three filter bank methods (WPT, EMD, and conventional Fourier filter bank, FFB) to enhance the quality of signal analysis, preserving the physical meaning of the extracted features. CSDI is applied to the acquisition cases of the acoustic, vibration, and combination of vibration and acoustic signals. Using a $k$-nearest neighbors algorithm, the classifier validation is carried out on the data measured in a turbine blade laboratory experiment and a laboratory test rig. Both setups are employed for simulating a set of multiple faults. Obtained results for classification accuracy show that the CSDI feature set, extracted from the considered components extracted by filter bank methods, leads to increasing the classification performance, mostly, in cases of low SNR for either case of acquired signal: acoustic or vibration. Moreover, the use of both data further improves the fault classification, resulting in a promising methodology to implement in CM of rotating machinery.

The agenda of this work is as follows: Section 2 summarizes the employed FBM as well as the compared similarity measures, including the introduced CSDI measure. Section 3 outlines the experimental setup used for the classifier validation carried out for two real-world databases of machine faults, holding acoustic and vibration recordings. Finally, discussion of the obtained results and regarded conclusions are given in Sections 4 and 5, respectively.

\section{Theoretical Background}

2.1. Filter Bank Methods. With the purpose of separating the information of spectral subbands, the filter bank methods (FBM) decompose bandwidth-limited signals into a set of narrow-band components. Thus, a given signal $x(t) \in \mathbb{R}(T)$ that has a finite bandwidth $\Delta F$ (with $F=[0,1 / 2 \Delta t]$, being $1 / \Delta t$ the sampling frequency) is decomposed into $M \in \mathbb{N}$ narrow-band components $\boldsymbol{\varphi}=\left\{\varphi_{m}(t): m \in M\right\}$ so that each one has a bandwidth $\Delta F_{m}$ such that $F_{m} \subseteq F$. Regarding acoustic and vibration analysis, the following approaches for FBM are widely used:

(i) Decomposition Using Fourier Filter Banks (FFB). Fourier transform, noted as $X(f)=\mathscr{F}\{x(t)\}$, provides a straightforward approach to implementing FBM so that several ideal bandpass filters are applied to the input signal $x(t) \in \mathbb{R}(T)$ in order to build a component set $\boldsymbol{\varphi}=\left\{\varphi_{m}(t): t \in T\right\}$ such that the support of $\varphi_{m}(f)$ equals the closure of the corresponding bandwidth, noted as $\bar{F}_{m}$. With the aim of obtaining an orthogonal FBM, all bandwidths are also required to be disjoint, that is, $F_{m} \cap F_{n}=\emptyset, \forall n \neq m$, with $n, m \in M$.

(ii) Wavelet Packet Transform (WPT). Wavelet Transform can be expressed in terms of the inner product between each basis $v_{j, k}^{n}(t) \in \mathbb{R}(T)$ and the considered signal $x(t)$. Thus, the wavelet coefficients $w_{j, n, k} \in$ $\mathbb{R}(T)$ are the inner product, $\left\langle x, v_{j, k}^{n}\right\rangle$, defined as $w_{j, n, k}=\int_{T} x(t) v_{j, k}^{n}(t) d t$, where $n \in \mathbb{N} \cup\{0\}$ is the oscillation parameter, and $j, k \in \mathbb{Z}$ are the scale and translation operation indexes, respectively. Therefore, a WPT basis function $v_{j, k}^{n}(t)$ is defined as $v_{j, k}^{n}(t)=$ $2^{j / 2} v^{n}\left(2^{j} t-k\right)$, where the initialization $(n=0)$ and remaining recursions $(n>1)$ are given in [14].

(iii) Empirical Mode Decomposition (EMD). This FBM is a self-adaptive time-frequency signal analysis method based on local characteristic time scales. EMD can decompose the complex and unstable signal into several nearly stable intrinsic mode functions (IMFs) as defined in [15]

2.2. Feature Estimation Based on Similarity Measures. With the aim of improving the discriminating ability of the feature set, we measure the similarity between the input signal $x(t)$ and each extracted $m$ th narrow-band component, $\varphi_{m}(t) \epsilon$ $\mathbb{R}(T)$, quantifying their mutual statistical dependence.

A straightforward approach to measuring the statistical similarity between two processes is their product moment, 


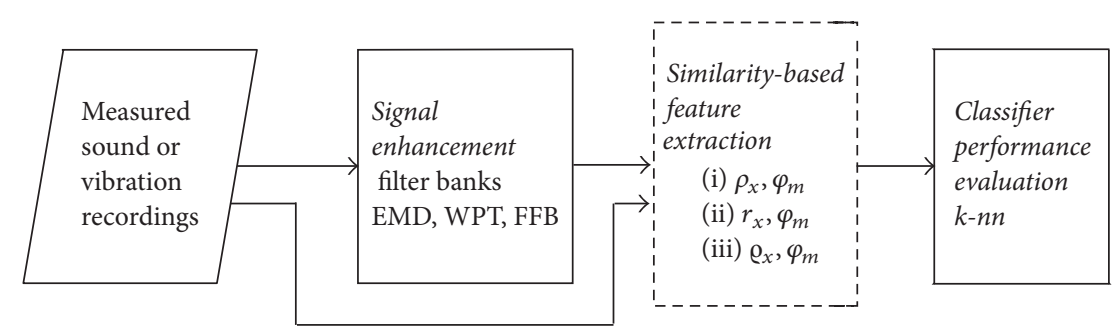

FIGURE 1: General scheme of the experimental setup.

termed Pearson's correlation coefficient $\rho_{x, \varphi_{m}} \in \mathbb{R}[-1,1]$, that quantifies the linear relationship of dependence as follows:

$$
\rho_{x, \varphi_{m}}=\frac{\mathbf{E}\left\{x(t) \varphi_{m}^{*}(t)\right\}}{\sigma_{x} \sigma_{\varphi_{m}}},
$$

where $\sigma_{\xi}^{2}=\mathbf{E}\left\{|\xi(t)|^{2}: \forall t \in T\right\}$ is the variance, with $\sigma_{\xi}^{2} \in \mathbb{R}^{+}$. Notations $\mathbf{E}\{\cdot\}$ and $(\cdot)^{*}$ stand for the expectation operator and conjugate, respectively. Note that both $x(t)$ and $\varphi_{m}(t)$ are assumed to be zero-mean stochastic process.

In a more elaborate approach, the statistical dependence between two processes can be estimated by using an information measure. In particular, we employ the Kullback-Leibler divergence (also termed relative entropy) that measures the difference between a couple of probability density functions, $p_{x}(u)$ and $p_{\varphi_{m}}(u)$, as follows:

$$
r_{x, \varphi_{m}}=\int_{T} p_{x}(u) \ln \left(\frac{p_{x}(u)}{p_{\varphi_{m}}(u)}\right) d u, \quad r \in \mathbb{R}^{+},
$$

where $u$ is an i.i.d. sample for each corresponding distribution. Note that $r_{x, \varphi_{m}}$ is not a distance in the formal sense since it is not symmetric and does not satisfy the triangle inequality. Still, it frequently serves as a similarity measure estimation between densities [16].

Nevertheless, either measure defined above, $r_{x, \varphi_{m}}$ or $\rho_{x, \varphi_{m}}$, assumes the stationarity of correlated processes. In practice, this assumption is far from being true for most of the acquired CM data. Besides, most of the state-of-the-art techniques for fault detection lie on spectral analysis of the measured signals. With the purpose of providing the analytical support for nonstationary processes and spectral significance, we introduce the similarity measure (termed cumulative spectral density index (CSDI)) that is built on the cross-correlation function $R_{x \varphi_{m}}(t, \tau):=\mathbf{E}\left\{x(t+\tau) \varphi_{m}^{*}(t)\right\}$ calculated between two second-order nonstationary processes.

Let $S_{x \varphi_{m}}(f)$ be the signal content distribution over the frequency domain to be represented through the crossspectral density between $\left\{x(t), \varphi_{m}(t)\right\}$ so that we compute the Fourier transform of the time-averaged function $R_{x \varphi_{m}}(t, \tau) \in$ $\mathbb{R}^{+}$as follows:

$$
S_{x \varphi_{m}}(f):=\int_{\mathbb{R}} \bar{R}_{x \varphi_{m}}(t) \exp ^{-j 2 \pi f t} d t
$$

where

$$
\bar{R}_{x \varphi_{m}}(\tau):=\lim _{T \rightarrow \infty} \frac{1}{T} \int_{-T / 2}^{T / 2} R_{x \varphi_{m}}(t, \tau) d t
$$

However, the complex-valued density $S_{x \varphi_{m}}(f) \in \mathbb{C}$ must be mapped into a real-valued domain. To this end, we apply the expected value of the squared modulus $\mathbf{E}\left\{|\cdot|^{2}\right\}$ as a positive semidefinite operator. Therefore, we obtain a measure of the spectral information shared between the signal $x(t)$ and its set of narrow-band components $\left\{\varphi_{m}(t): \forall m \in M\right\}$.

Nonetheless, the spectral density can hold powerful components (i.e., that are locally concentrated), strongly biasing the expectation operator estimator [17]. To overcome this issue, we propose to introduce the expected value over the cumulative energy function in the CSDI definition as follows:

$$
\varrho_{x, \varphi_{m}}=\mathbf{E}\left\{\int_{0}^{f}\left|S_{x \varphi_{m}}(\tilde{f})\right|^{2} d \tilde{f}: \forall f \in F_{m}\right\} .
$$

Note that the higher the value of similarity for $\rho_{x, \varphi_{m}} \in \mathbb{R}^{+}$ or $\varrho_{x, \varphi_{m}} \in \mathbb{R}^{+}$, the higher the statistical association between variables. By contrast, lower values of $r_{x, \varphi_{m}}$ imply a close relationship since it can be explained as the distance between both compared probability density functions.

\section{Experimental Setup}

As shown in Figure 1, the presented fault detection methodology using acoustic and vibration signals comprises the following stages: (i) signal enhancement, applying one of the examined filter bank decompositions (EMD, WPT, or FFB), (ii) feature extraction, testing each of the proposed similarity measures (Pearson's correlation coefficient, crossentropy spectral analysis, and cumulative spectral density index) between the raw measured data and its decomposed narrow-band components, and (iii) classifier performance validation, feeding the extracted feature set into the classification algorithm.

Besides, we employ a leave-one-out classification scheme at different signal-to-noise ratio levels to validate the robustness and accuracy of the proposed methodology. Since our contribution focuses more on the feature extraction stage, we employ the $k$-nn algorithm as a simple classifier. Several reasons account for the widespread use of this classifier: it is straightforward to implement, it leads to a reliable recognition performance (thanks to the nonlinearity of its decision boundaries), and its complexity is assumed to be independent of the number of classes. So, the tuning of the $k$-nn classifier is carried out by the thumb rule (that is, the square root of the number of instances that each class holds). 


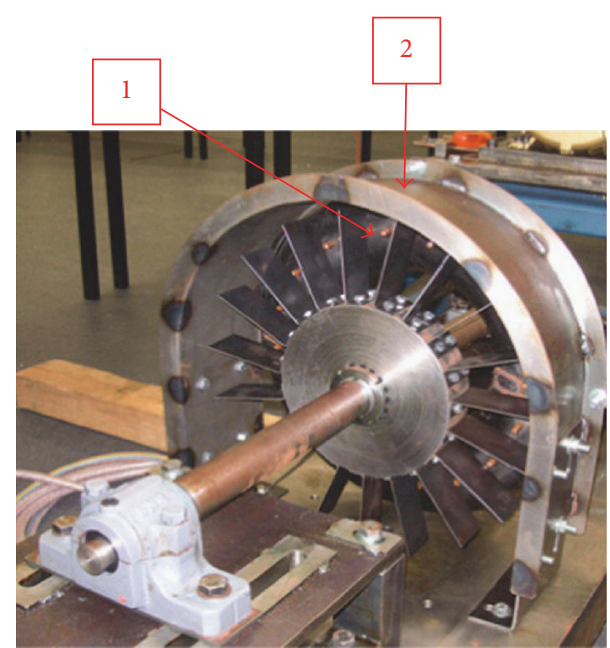

FIGURE 2: Experimental test rig equipped with 19 blades. (1) Six air jets located on a toroidal ring that is supplied with high pressure air and (2) location of microphone and accelerometer mounting [18].

The methodology is tested using the collected data from two different laboratory applications: a turbine blade degradation and a rotating system with rolling element bearing and shaft defects. Here, training is carried out for each source of measured data: acoustic and vibration signals. We also consider validation in the case of multiple failures (in particular, shaft and bearing defects) a challenging case of discrimination. For this task, the combination of both acquired signals (acoustic and vibration) is considered in order to increase the classification accuracy.

3.1. Validation on Damage Detection of Turbine Blades. In this experiment, we validate the performance methodology using just acoustic signals to identify degradation faults of rotating machines. In particular, we use a database holding the acoustic recordings acquired from a damage of turbine blades, provided by [18]. The test rig consisted of a nineteenflat bladed disk arrangement, where each blade is $1.2 \mathrm{~mm}$ thickness. The experimental system was driven by an electric motor running at $2000 \mathrm{rpm}$. With the aim to generate the external forces that excite the blades at multiple shaft speeds with significant turbulent components, a toroidal ring with six air jets was located in front of the blade disk emitting highvelocity air. Figure 2 presents the test rig without the external protective housing.

For the sake of simulating the potential degradation, one blade was replaced by a thinner blade of $0.9 \mathrm{~mm}$ thickness, reducing the structural stiffness. So, two different measurements were recorded simultaneously: (i) acoustic signals from a microphone flush mounted on the inner casing surface and (ii) mechanical vibration signal by an accelerometer located in the vertical plane of casing surface. All recordings that lasted ten seconds were acquired at $65536 \mathrm{~Hz}$ sampling frequency, considering three conditions: air jets turned off (no blade vibration), air jets on (all blades of the same vibration), and air jets on with a simulated broken blade as detailed in [18].

Due to the fact that useful bandwidth was assumed at $8.192 \mathrm{kHz}$, all acoustic signals were filtered by a low-pass Butterworth filter with a cut frequency at $3500 \mathrm{~Hz}$ and then downsampled to 8192 samples per second, allowing reducing the computational burden. As to obtaining several trajectories, each preprocessed recording was split into shorter segments, each one lasting one second. For every considered fault condition, Figure 3 displays some examples of the segmented recordings together with their time-frequency domain computed by Short-Time Fourier Transform (STFT) with 2048 frequency bins and the Hamming window with 256 samples and $50 \%$ overlapping.

After a single visual inspection of Figure 3, some remarks can be highlighted regarding the distribution of information on narrow frequency bands. Thus, the vibration signal (when the air jets are turned off) indeed manifests several spectral components under $1000 \mathrm{~Hz}$, and there is not any information above, which implies that the turbine blades provide the vibration. At the same time, the acoustic signal shows several fading harmonics of the turbine blade fundamental frequency at $633.3 \mathrm{~Hz}$, providing better information about the turbine blade rotation. On the other hand, the vibration signal clearly shows a difference between the undamaged and damaged conditions when the air jets are turned on. In fact, the harmonic with the highest amplitude switches from the first to the fifth harmonic. By contrast, the acoustic signal does not provide any visual distinction between both turbine blade conditions inasmuch as all harmonics have similar amplitudes. Therefore, a more detailed analysis of narrow spectral bands should be carried out to explain the degradation faults observed for rotating machines.

3.1.1. Signal Enhancement Using Filter Bank Methods. We apply the considered FBM to the acoustic and vibration signals using the following setup:

$F F B$ : the number of nonoverlapping narrow-bands $F_{m}$ is adjusted to $M=8$, where each one has the same bandwidth $\Delta F_{m_{1}}=\Delta F_{m_{2}} \forall m_{1}, m_{2} \leq M$, as suggested in [19].

WPT: we perform this decomposition with near optimal time-frequency localization using the Daubechies 4 function (DB4) (with three decomposition levels) as mother wavelet $\psi(t)$ as carried out in [20]. Since the wavelet packet-basis function $v_{j, k}^{n}(t)$ is iteratively calculated as $v_{j, k}^{n}(t)=2^{j / 2} v^{n}\left(2^{j} t-k\right)$, the initialization $(n=0)$ and remaining recursions $(n>1)$ are done as in [14].

$E M D$ : even that the EMD approach does not require any parameters to adjust, we still use only the first 8 IMFs due to the set $\varphi$ should have the same cardinal for all the FBM; that is, $M=8$. The following iterative procedure implements the EMD procedure:

(1) Compute the interpolation function, $\Xi\{\cdot, \cdot\}(t)$ (ensuring the existence of its derivative) 

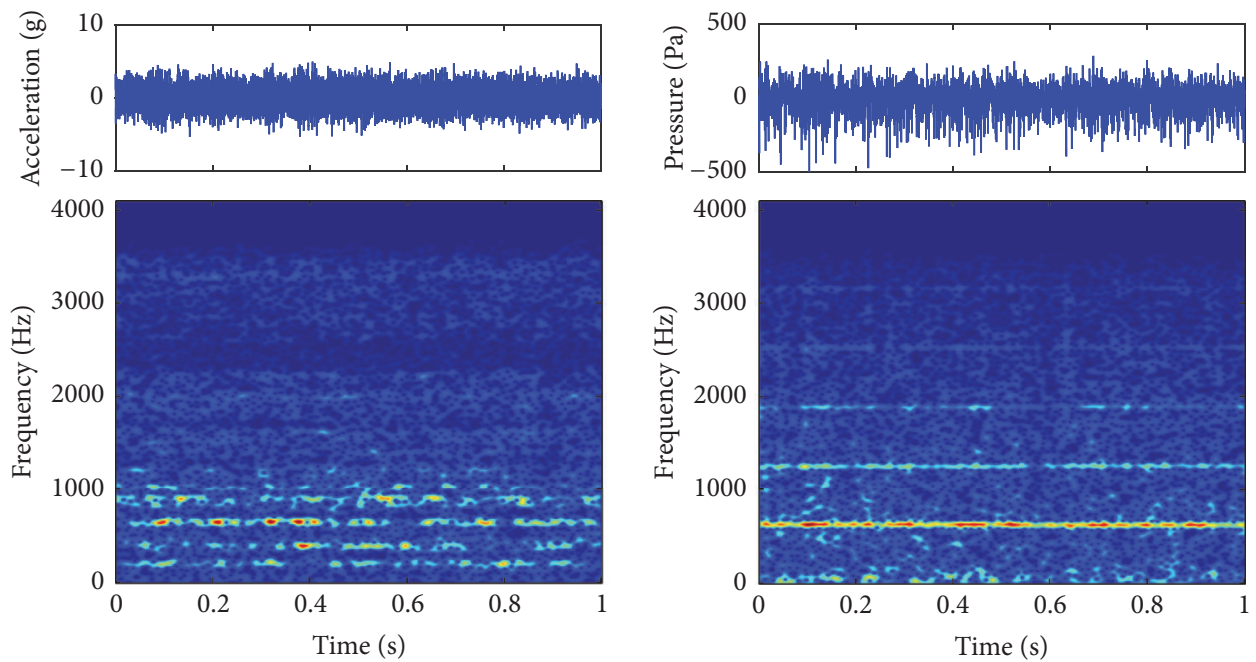

(a) Undamaged with air jets not turned on
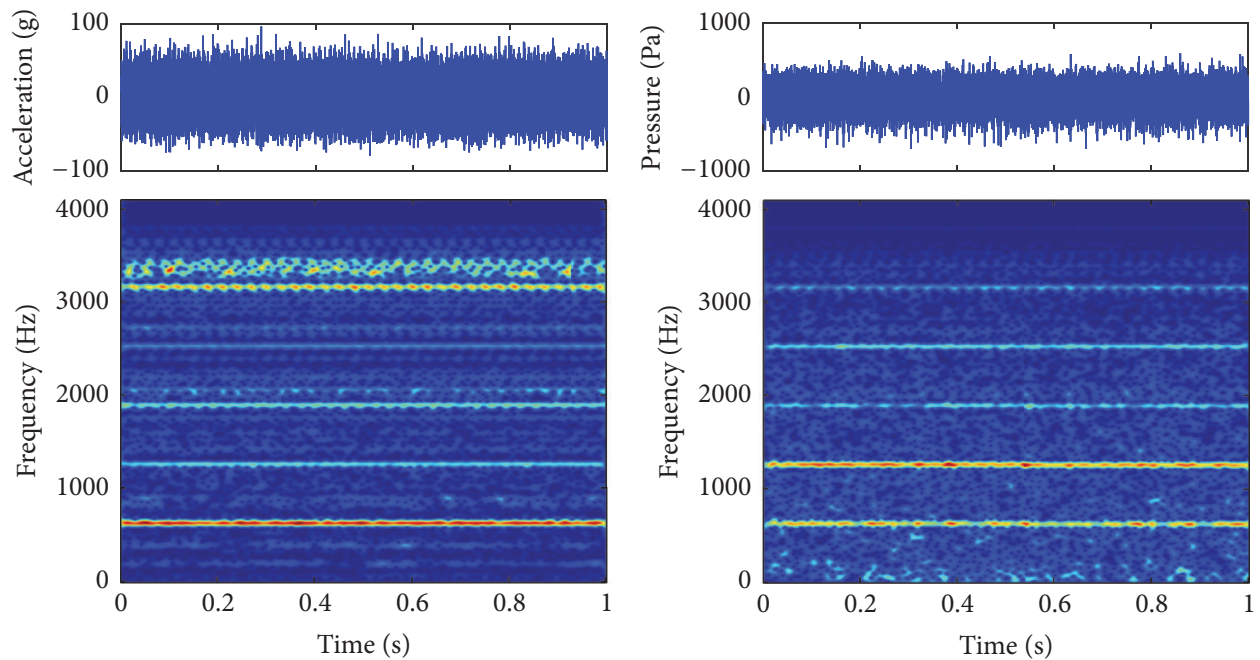

(b) Undamaged with air jets on
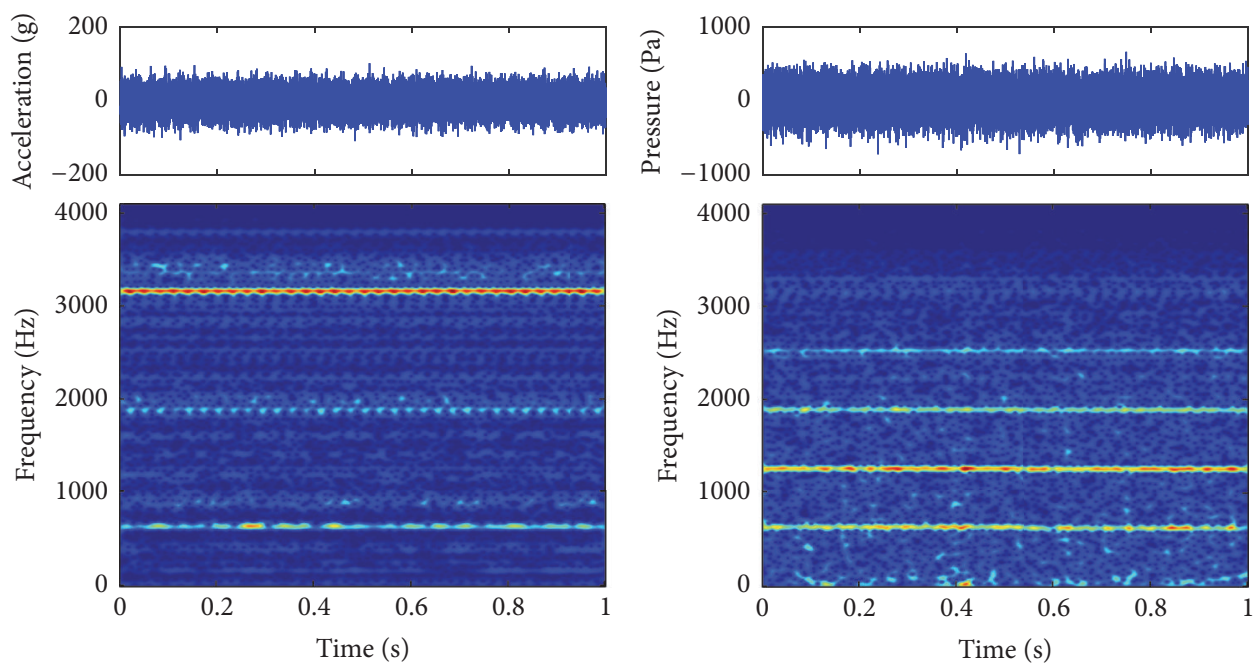

(c) Damaged with air jets on

FIGURE 3: Exemplary of time-frequency representation estimated for wind turbine vibration (left) and acoustic (right) signals with different conditions. 

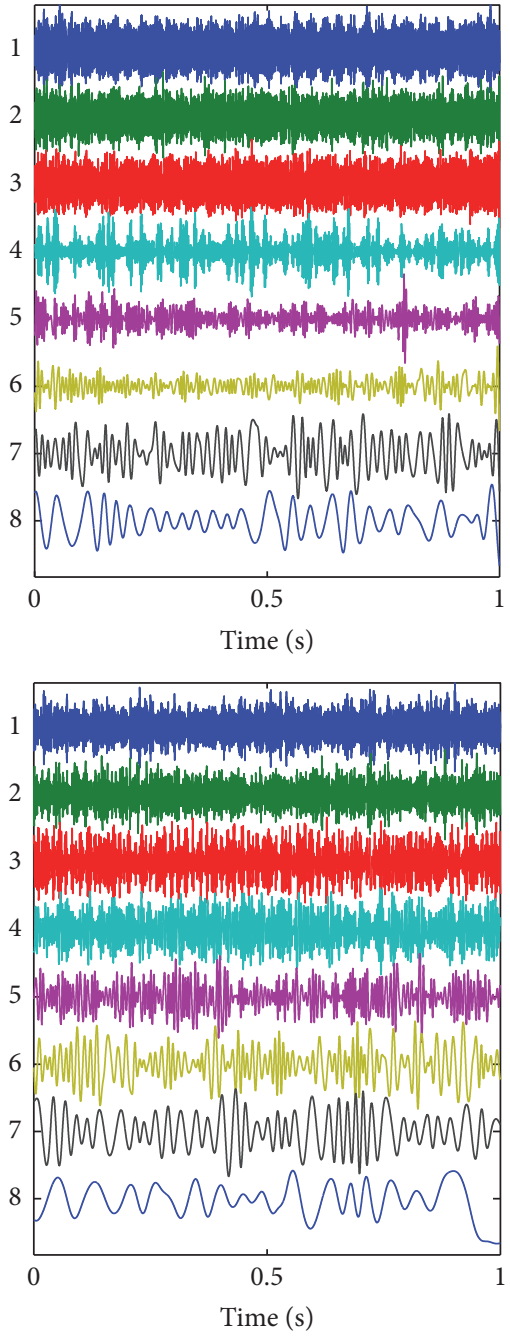

(a) EMD
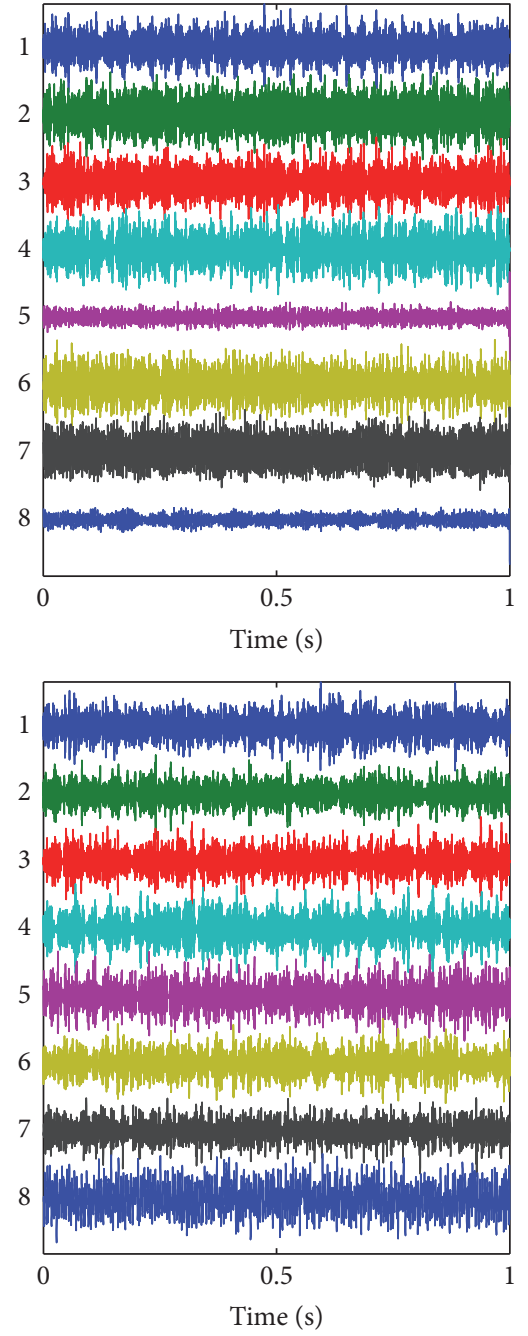

(b) WPT
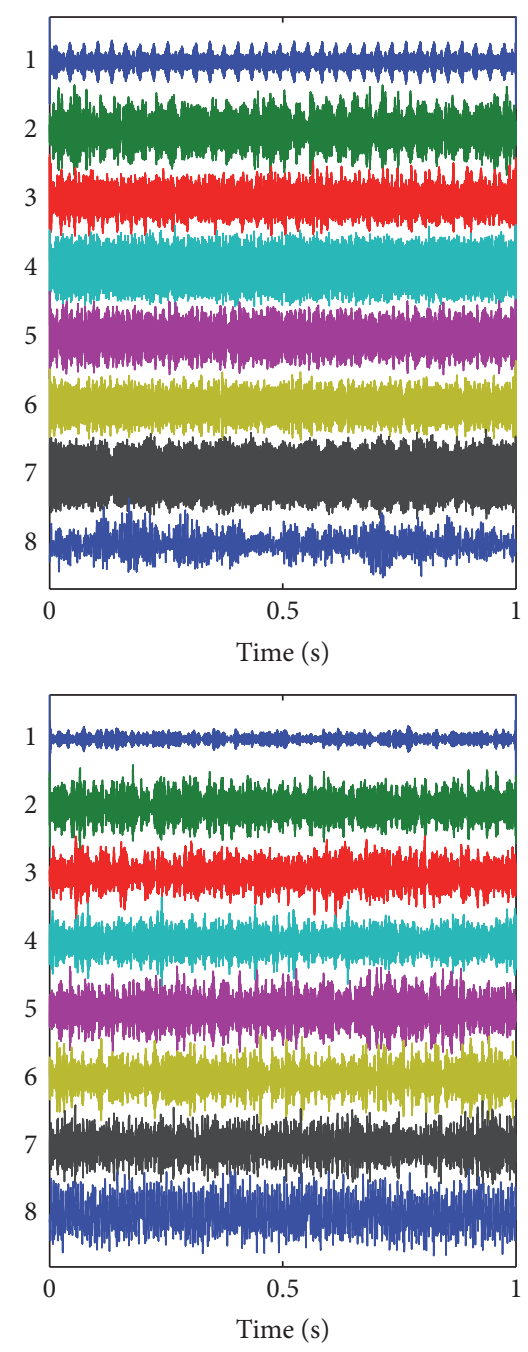

(c) FFB

FIGURE 4: Exemplary of computed narrow-band decompositions for a wind turbine under undamaged condition with air jets on for vibration (top row) and acoustic (bottom row). Note that the smaller the cardinal of narrow-bandwidth, the higher its spectral content.

between local minimal and local maximal points of $x(t)$, yielding, respectively, the sets:

$$
\begin{aligned}
& l_{\text {min }}:=\{t: \dot{x}(t)=0 \wedge \ddot{x}(t)>0\}, \\
& l_{\text {max }}:=\{t: \dot{x}(t)=0 \wedge \ddot{x}(t)<0\} .
\end{aligned}
$$

As a result, the inferior and superior envelopes are obtained:

$$
\begin{aligned}
& \chi_{\text {min }}(t)=\Xi\left\{l_{\text {min }}, x\left(l_{\text {min }}\right)\right\}(t), \\
& \chi_{\text {max }}(t)=\Xi\left\{l_{\text {max }}, x\left(l_{\text {max }}\right)\right\}(t) .
\end{aligned}
$$

(2) Compute the average $\bar{\chi}(t)=\left(\chi_{\min }(t)+\right.$ $\left.\chi_{\max }(t)\right) / 2$.

(3) Extract the IMF $\varphi_{m}(t)=x(t)-\bar{\chi}(t)$.

(4) If the stop criterion is fulfilled $m=m+1$; make $x(t)=\varphi_{m}(t)$, and return to the step one.

(5) Make $\phi(t)=\varphi_{M}(t)$; exit.
Steps (1) to (4) are performed over again until $\varphi_{m}(t)$ is nonconvex. Otherwise, the last step is applied.

Figure 4 displays an example of the decompositions performed for vibration (top row) and acoustic (bottom row) data.

3.1.2. Feature Extraction and Classification. In order to discriminate the fault classes properly, the feature set is extracted for each considered measure of similarity that is estimated between the extracted narrow-band component and measured signal. Therefore, each measure yields a single feature set $\xi \in \mathbb{R}^{n \times M}$, holding $n$ observations and $M$ characteristics, that is further fed into the classifier under leave-one-out classification scheme. By the thumb rule, we adjust the number of neighbors as five for the used $k$-nearest neighbor classifier.

Concerning simulation of a noisy real-world environment, the classifier performance is evaluated for several SNR 

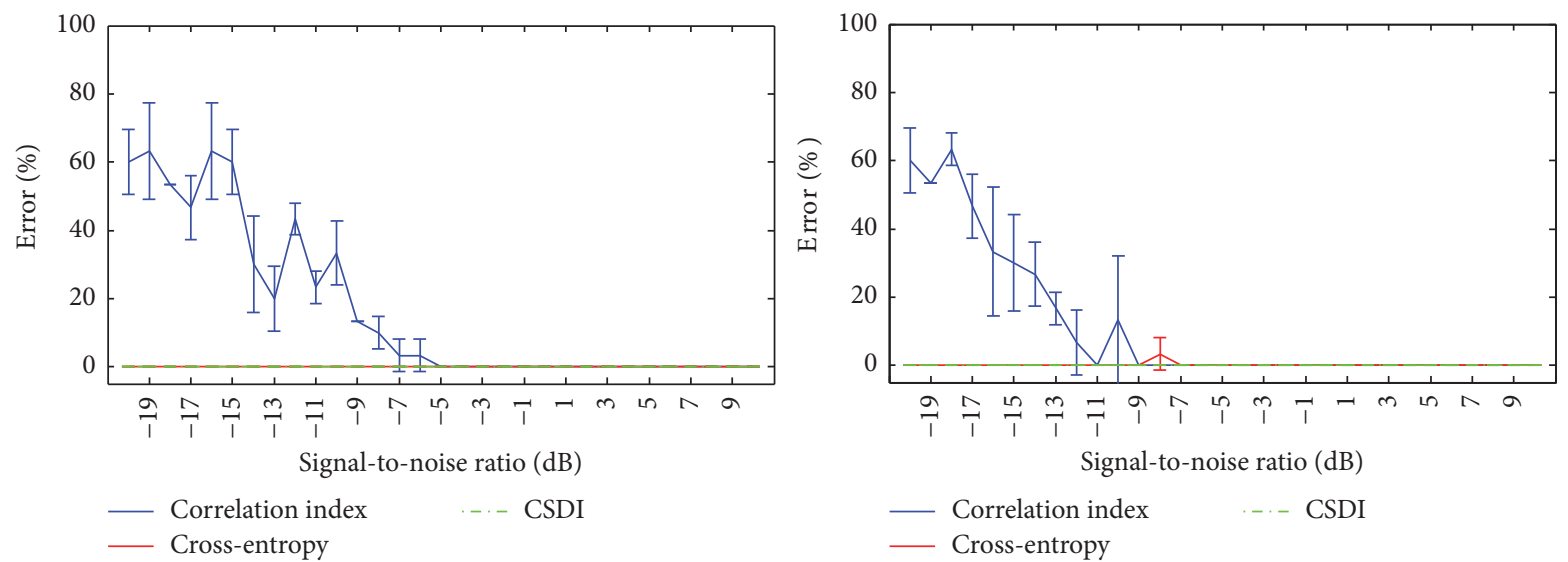

(a) EMD
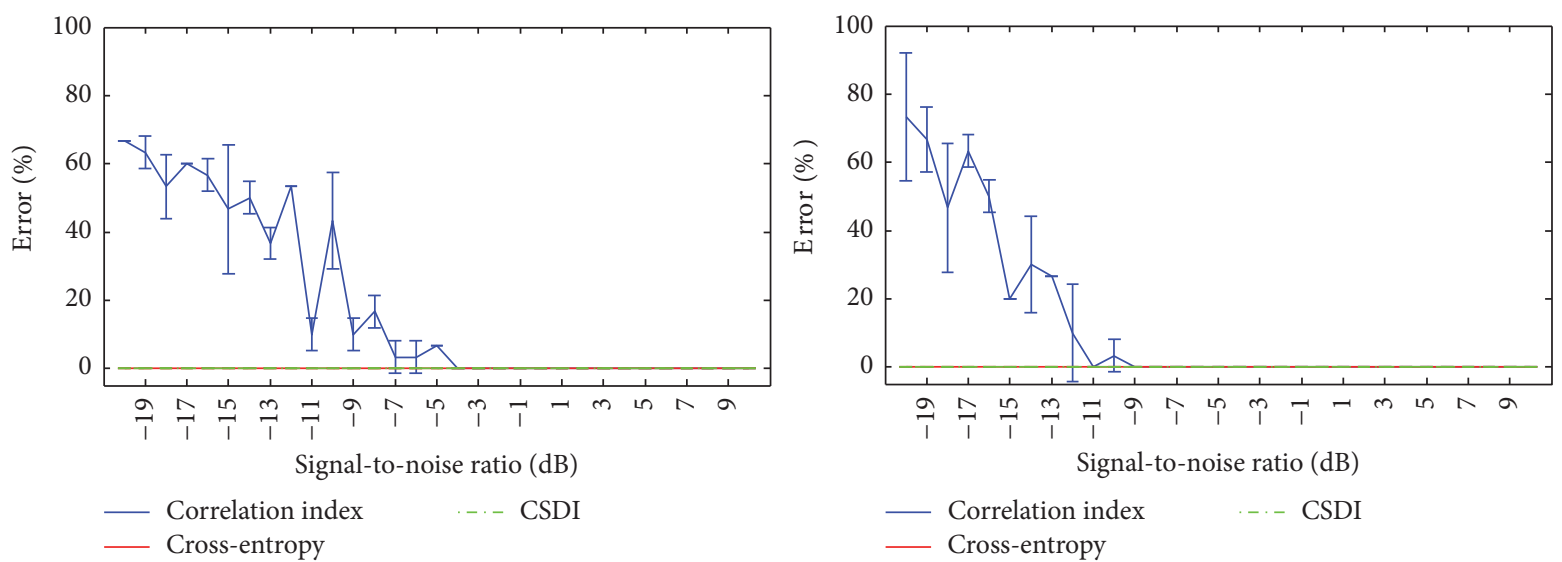

(b) WPT
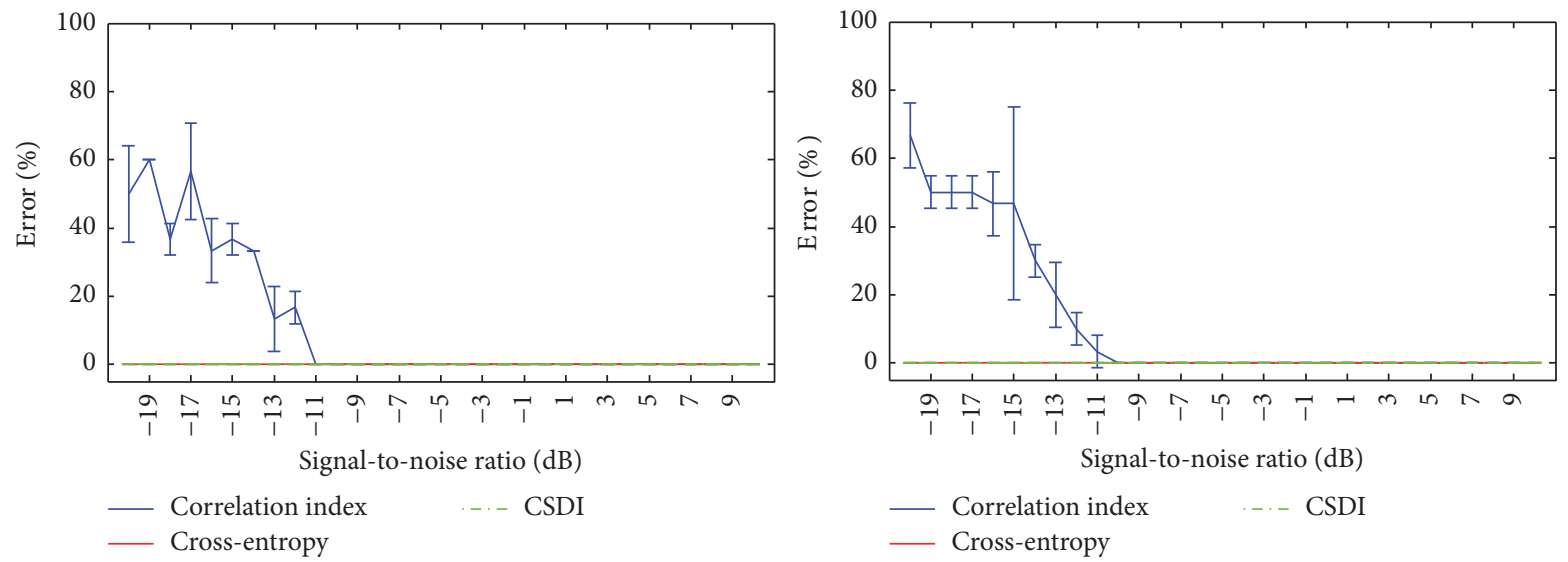

(c) FFB

FIGURE 5: Obtained classification error by the pair of filter bank methods and similarity measures with respect to different SNR levels of raw vibration (left) and acoustic (right) signals from turbine blade experiment.

levels, ranging from $-20 \mathrm{~dB}$ to $10 \mathrm{~dB}$. Figure 5 shows the classification error performed by each filter bank method for the vibration (left column) and acoustic (right column) signals. Regardless of the considered SNR, every considered FBM in combination with either the cross-entropy or CSDI measure enables performing the lowest possible error of classification. By contrast, the correlation index performs the worst when the SNR levels are lower than $-3 \mathrm{~dB}$.

3.2. Experiment on Bearing Test Rig. The methodology is also validated on the bearing test rig presented in Figure 6. The mechanical system consists of a shaft driven by a $1.5 \mathrm{HP}$ 


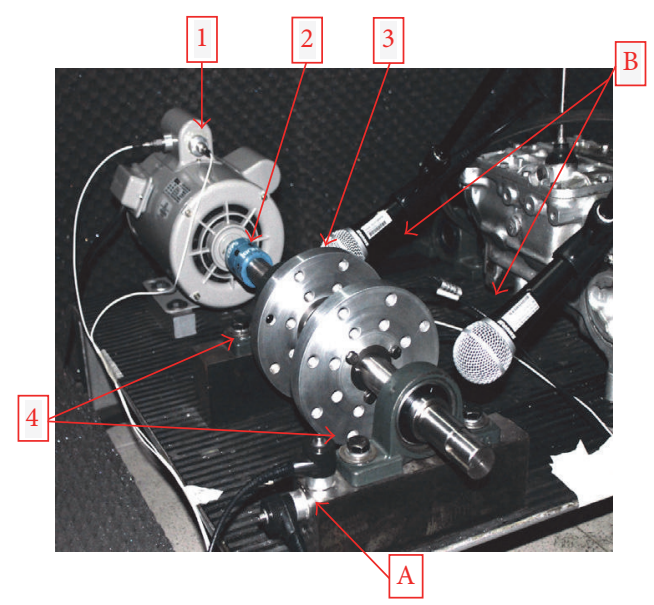

FIGURE 6: Experimental test rig used for experimentation: (1) motor driven, (2) rigid coupling, (3) drilling wheels, (4) bearing housing. Sensors location: (A) accelerometers and (B) microphones.

DC electric motor that is able to reach $1720 \mathrm{rpm}$ through the equipped rigid coupling. We consider the following fault classes in rotating machines, three bearing faults, that is, inner race (noted as BPFI), outer race (BPFIO), and ball elements (BSF), and two shaft faults, unbalance (UNB) and misalignment (MIS).

The employed database holds 40 acoustic recordings lasting three seconds at $44.1 \mathrm{kHz}$ sampling frequency and 40 vibration recordings acquired simultaneously, lasting four seconds at $25.6 \mathrm{kHz}$ sampling rate. The acoustic signals were acquired by two microphones located at a distance of $2 \mathrm{~cm}$ in front of each bearing housing. Simultaneously, the vibration recordings were collected in the horizontal plane, employing several accelerometers mounted on bearing supports since the simulated damage was placed at the shaft end. Furthermore, the signals were downsampled eight times at the sampling frequency of $3200 \mathrm{~Hz}$ and $5512 \mathrm{~Hz}$ for the vibration and acoustic recordings, respectively. Figures 7 and 8 display some examples of the time-frequency representation estimated for several bearing faults (namely, outer race, inner race, and ball element) together with the undamaged condition. Note that each fault gathers energy over narrow bands regardless of the type of acquired signal: vibration (see Figure 7) or acoustic (Figure 8). Besides, the electromotor introduces to the vibration signals a powerful interference at $120 \mathrm{~Hz}$, while the influence of the electric interference on the acoustic data is very weak.

As in the above-examined database, the FBM approaches are implemented, fixing $M=8$ as the number of considered narrow subbands. Figure 9 shows some examples of the performed signal enhancement for undamaged (Figure 9(a)), unbalance (Figure 9(b)), and outer race bearing (Figure 9(c)) conditions. All plots are normalized for the sake of better visual comparison. As seen, the signal enhancement provided by WPT allows visually distinguishing the outer race defect in the high-frequency narrow-band components since those components exhibit the impulsive behavior that characterizes this type of damage. Nonetheless, it is hard to infer some
TABLE 1: Best classification error obtained by means of each pair filter bank method, similarity measures compared with statistic features, using vibration (upper part), acoustic (middle part), and combined vibroacoustic (lower part) signals with $\mathrm{SNR}=-20 \mathrm{~dB}$ from bearing fault test rig experiment.

\begin{tabular}{lccc}
\hline Similarity measure & EMD & WPT & FFB \\
\hline Correlation & $83.3 \pm 0.81$ & $82.9 \pm 0.45$ & $83.3 \pm 0.37$ \\
Cross-entropy & $14.6 \pm 0.37$ & $11.2 \pm 0.2$ & $10 \pm 0.2$ \\
CSDI & $11.2 \pm 0$ & $\mathbf{9 . 2} \pm \mathbf{0 . 2}$ & $9.6 \pm 0.2$ \\
Statistics & $85 \pm 0.8^{(7)}$ & $85 \pm 0.8^{(8)}$ & $85 \pm 0.2^{(8)}$ \\
\hline Correlation & $85.8 \pm 0.29$ & $81.7 \pm 0.68$ & $84.6 \pm 0.23$ \\
Cross-entropy & $23.3 \pm 0.68$ & $\mathbf{2 3 . 7} \pm \mathbf{0 . 2 3}$ & $24.7 \pm 0.42$ \\
CSDI & $27.9 \pm 0.54$ & $24.2 \pm 0.19$ & $27.1 \pm 0.35$ \\
Statistics & $85 \pm 0.68^{(7)}$ & $83.3 \pm 0.29^{(8)}$ & $85 \pm 0.29^{(8)}$ \\
\hline Correlation & $83.3 \pm 15.3$ & $82.5 \pm 12.65$ & $83.3 \pm 15.29$ \\
Cross-entropy & $7.5 \pm 7.4$ & $10 \pm 6.84$ & $7.5 \pm 7.4$ \\
CSDI & $\mathbf{4 . 2} \pm \mathbf{3 . 7}$ & $\mathbf{4 . 2} \pm \mathbf{3 . 7}$ & $7.5 \pm 7.4$ \\
Statistics & $84.2 \pm 13.75^{(7)}$ & $82.9 \pm 12.65^{(8)}$ & $84.2 \pm 15.29^{(8)}$ \\
\hline
\end{tabular}

information correctly to identify each considered machine failure in each narrow-band component waveform. Note that the $k$-nn classifier is employed with five neighbors, using a leave-one-out classification scheme.

Furthermore, Figure 10 shows that each considered similarity measure produces a feature set that provides an equivalent classifier accuracy at the very high SNR (beyond $9 \mathrm{~dB}$ ) regardless of the used FBM signal analysis. However, the acoustic analysis performs worse when the SNR level decreases, so that, at the very low values of SNR, the features using CSDI and cross-entropy outperform clearly the baseline Pearson's correlation coefficient. Besides, the combination of acoustic and vibration signals decreases the achieved classification error for the SNR values under $-5 \mathrm{~dB}$.

With the purpose of determining the best combination of FBM and similarity measure, we consider the results performed at $-20 \mathrm{~dB}$ SNR level since it is the worst tested scenario when noise highly contaminates the input. Thus, Table 1 summarizes the best results for the vibration (upper part), acoustic (middle part), and combined vibroacoustic analysis (lower part). As seen, either measure (cross-entropy or CSDI) can reach the highest performance using a single type of data, vibration or acoustic.

Another aspect of training to consider is the classification strategy for the case of combining both types of data simultaneously. For the sake of simplicity, we consider a stacked combination of classifiers (namely, product combiner), yielding a notably raising of the performed accuracy. Note that this improvement holds just for very low SNR values. Otherwise, every considered measure performs well. For the sake of comparison, the classification performance is computed by 24 traditional statistic features such as mean, standard deviation, and skewness [21]. From each narrow-band component, the statistics features are estimated, and then, the components are added successively until we obtain the best accuracy (the superscript indicates the number of considered components). 


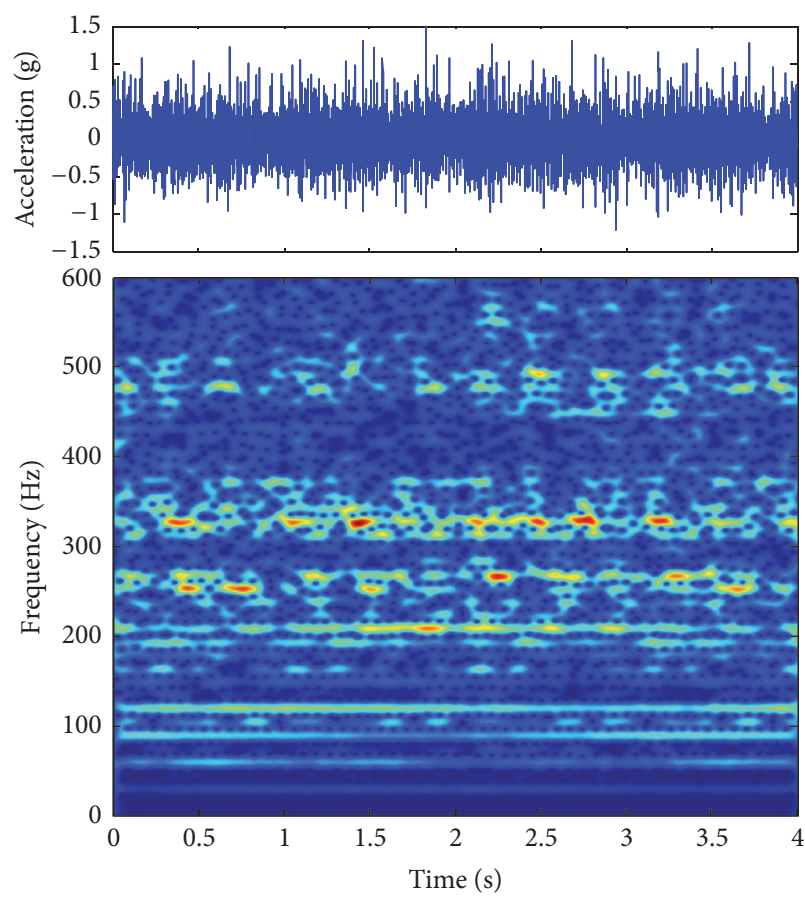

(a) Undamaged
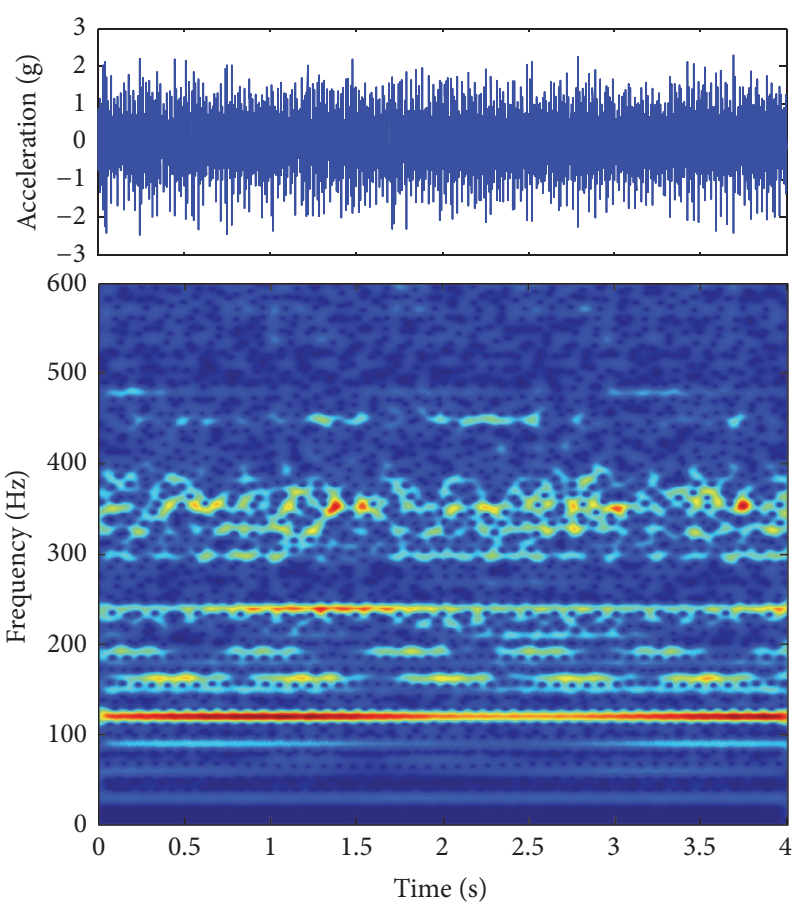

(c) Inner race bearing
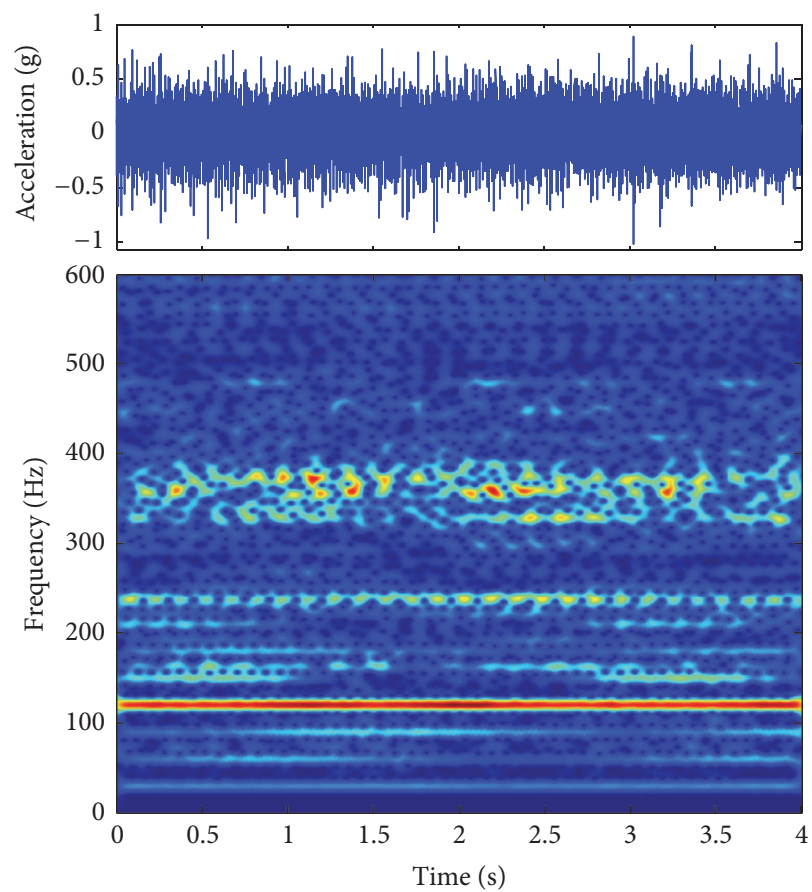

(b) Outer race bearing
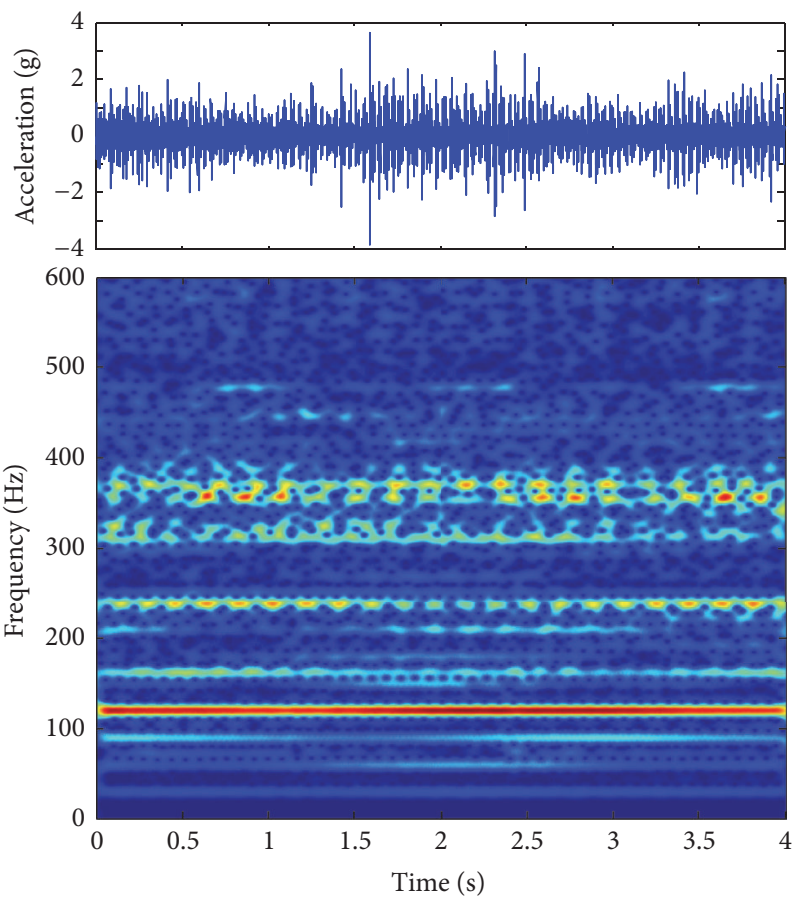

(d) Ball bearing

FIGURE 7: Vibration signals. Time-frequency representation for the fault conditions simulated at laboratory test rig.

As a result, the proposed methodology outperforms the classical statistics commonly used to characterize the machine faults.

In the multiclass problem, the confusion matrix is included (see Figure 11) for each type of signal analysis, providing more information regarding the classification errors of other fault types. The matrices, estimated for the worst tested scenario (i.e., $-20 \mathrm{~dB}$ ), show the number of misclassified instances of each considered fault. As seen, the acoustic analysis achieves the lowest accuracy, especially, in the cases of discriminating between classes: BSF and UND, BSFMIS, and MIS-UNB. Also, the vibration analysis performs 


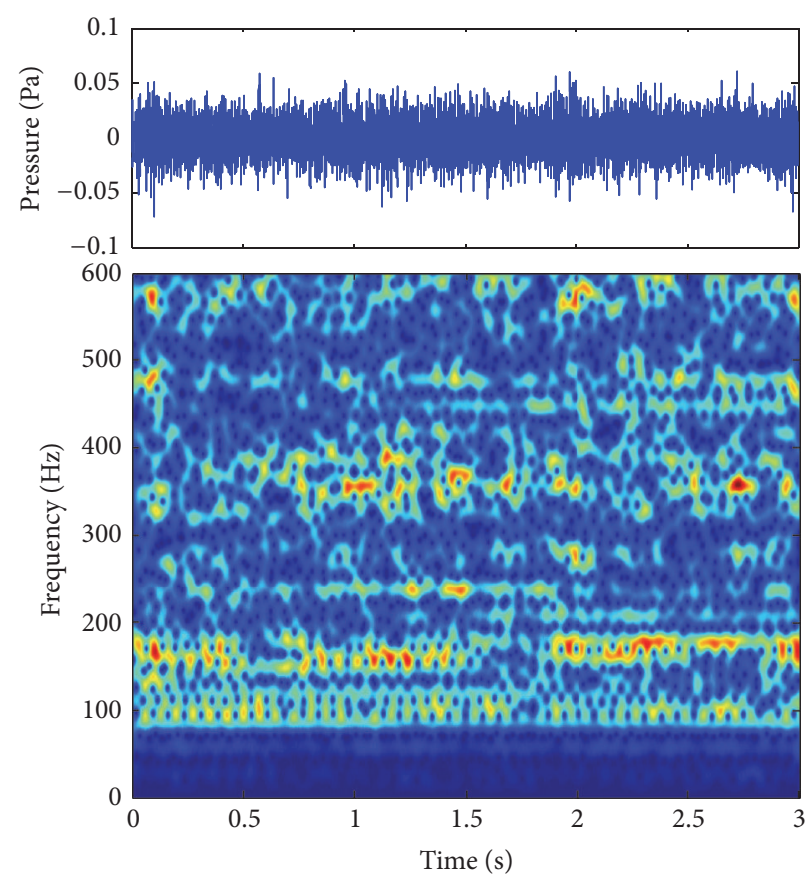

(a) Undamaged
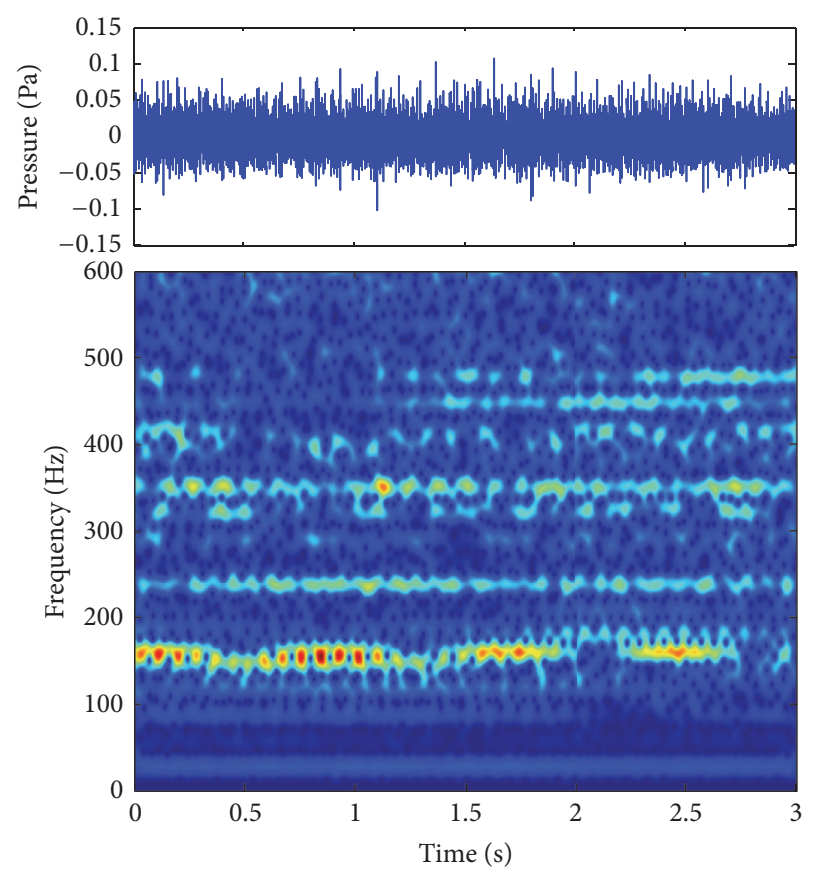

(c) Inner race bearing

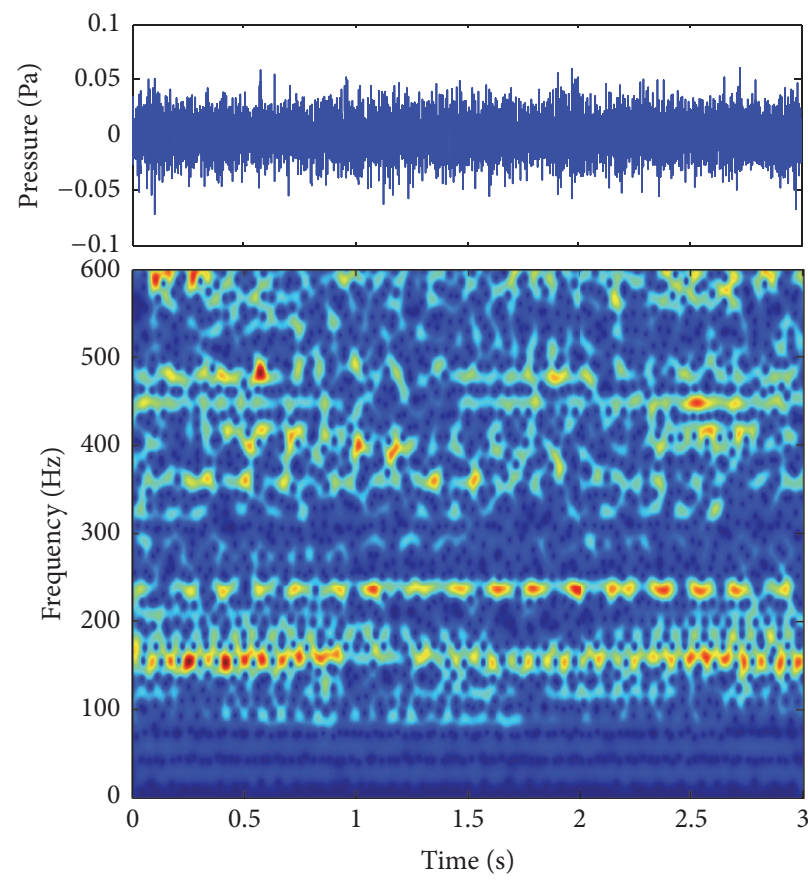

(b) Outer race bearing

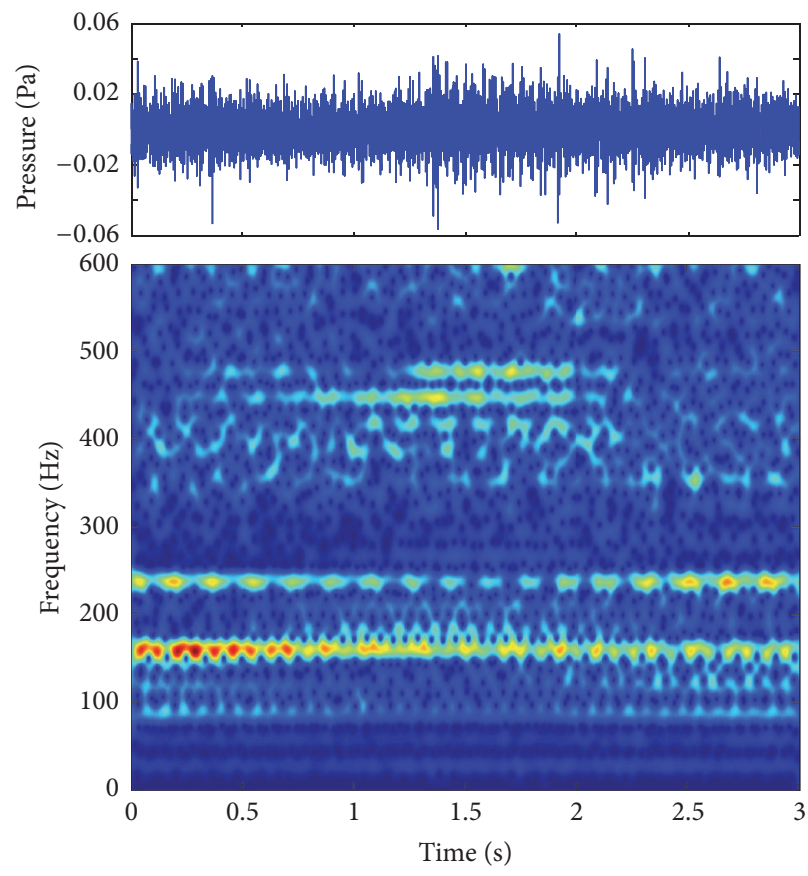

(d) Ball bearing

Figure 8: Acoustic signals. Time-frequency representation for the fault conditions simulated at laboratory test rig.

better though some degradation appears for discriminating between the classes BPFI and BSF.

The next aspect to consider is the influence of each similarity measure. In particular, Pearson's correlation coefficient worsens the classifier performance. However, the combination of acoustic and vibration analysis allows obtaining the highest multiclass accuracy, also improving the accuracy provided by each similarity measure.

\section{Discussion}

In this work, the proposed methodology for fault detection aims to enhance the acoustic and vibration analysis by introducing the cumulative spectral density index that is applied to the extracted spectral components with narrow bandwidth. However, some key points regarding the used FBM and similarity-based features are discussed as follows: 

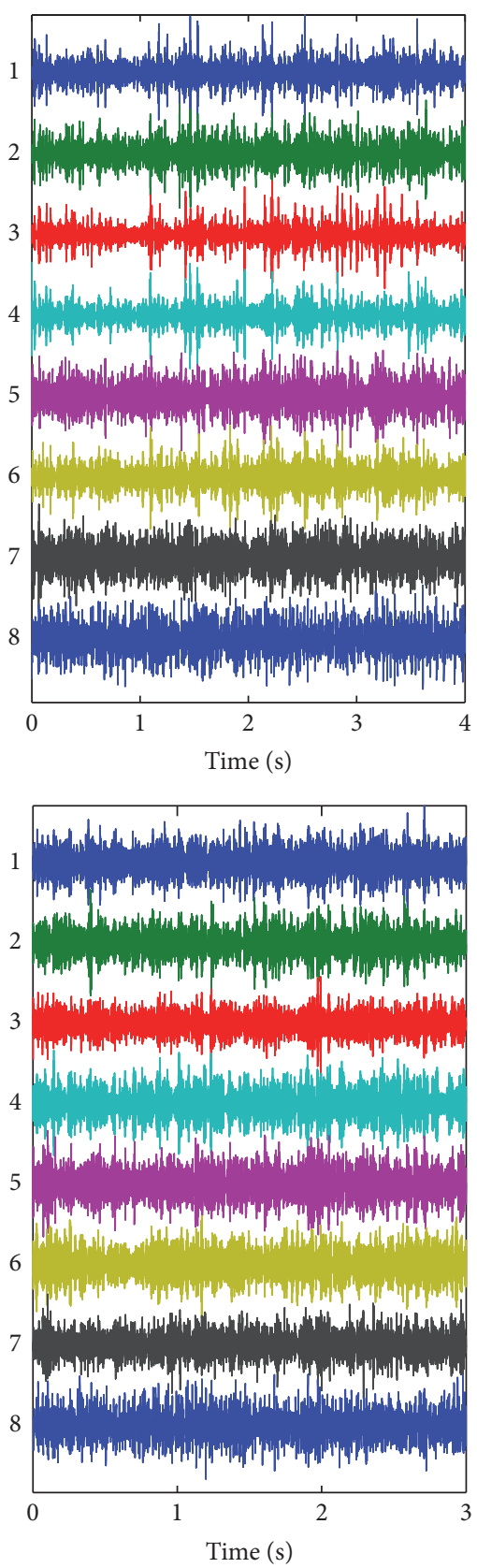

(a) Undamaged
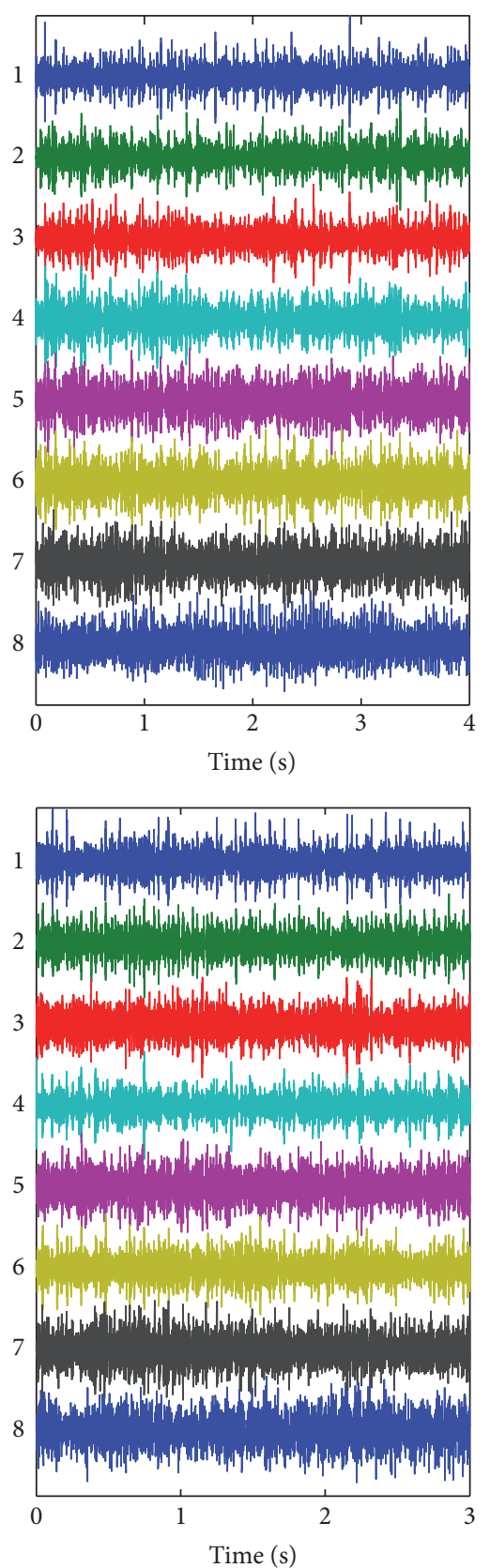

(b) Unbalance
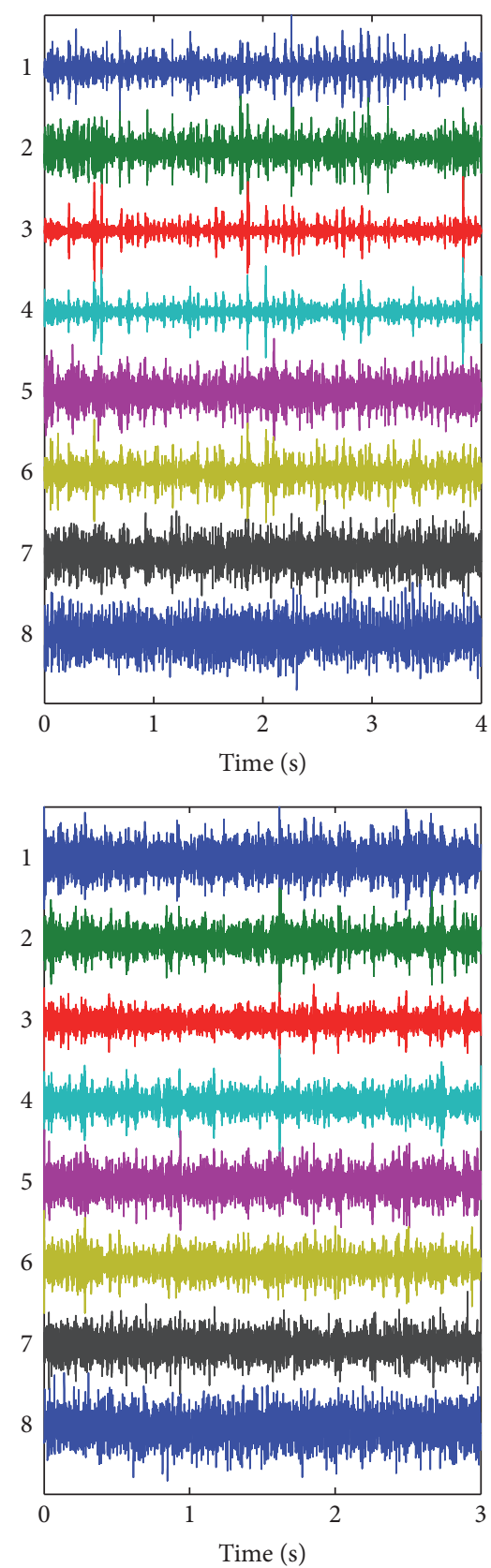

(c) Outer race bearing

FIgURE 9: Exemplary WPT decomposition of test rig for vibration (top) and acoustic (bottom) signals. Note that the smaller the cardinal of narrow-bandwidth, the higher its spectral content.

(i) Although this work considers signals acquired from machines at constant speed, it is possible to find stationary or nonstationary behaviors, generated by machine faults or external interferences. However, the vibration and acoustic signals are differently influenced by these external factors. Thus, the vibration signal displays a stationary component associated with an electric motor disturbance that hides the bearing frequency fault due to its high energy (see Figure 7). By contrast, in the acoustic signal, the same external disturbance shows a nonstationary behavior that is similar to the bearing faults (see Figure 8). Therefore, the latter signals are more vulnerable to external interferences than the vibration signals.

(ii) With the aim of improving the discriminating ability of the feature set, we test three measures of similarity between the input signal and each extracted narrowband component. Specifically, Pearson correlation index performs well in cases of large SNR values 

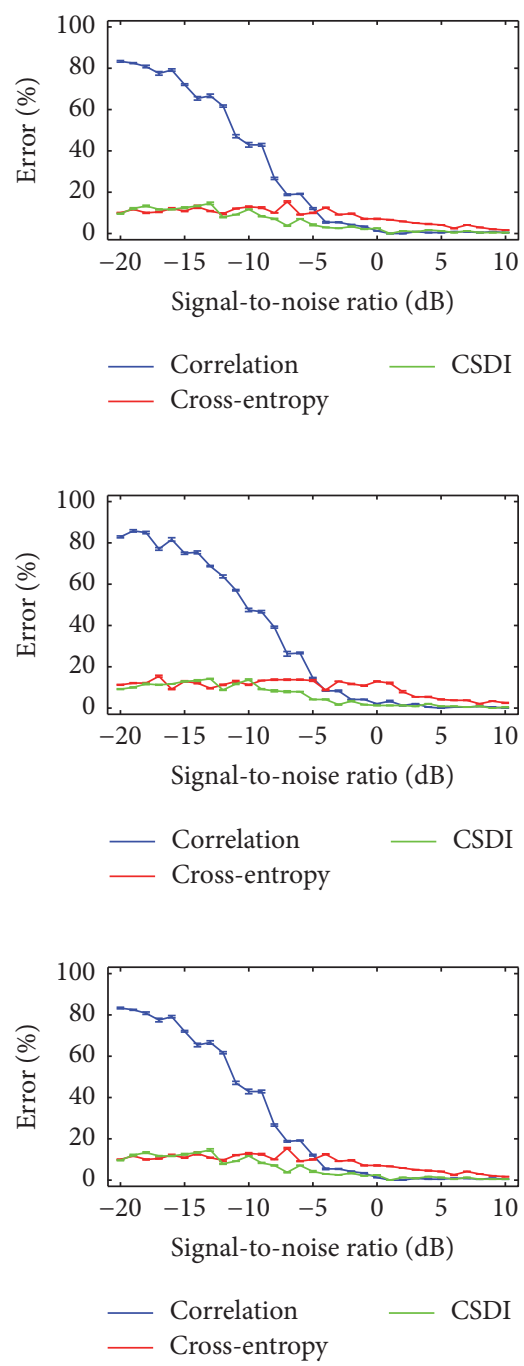

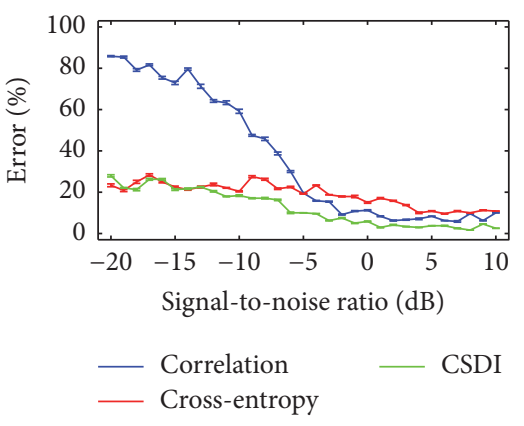

(a) EMD

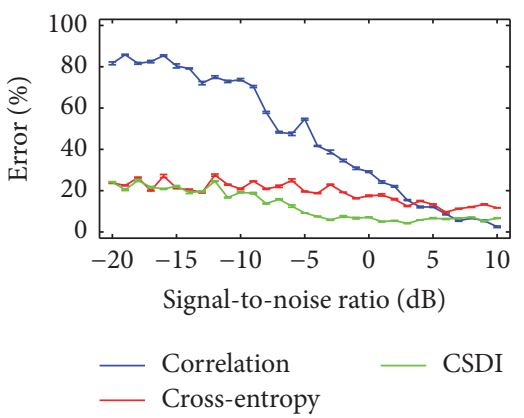

(b) WPT

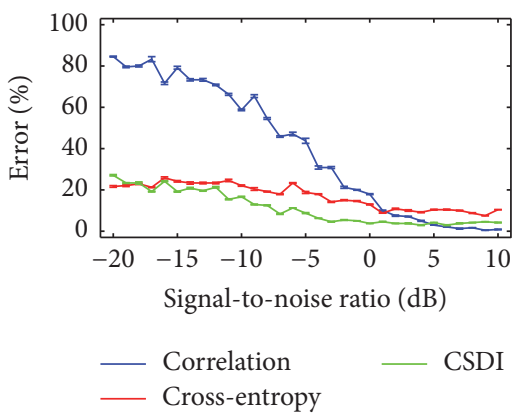

(c) FFB
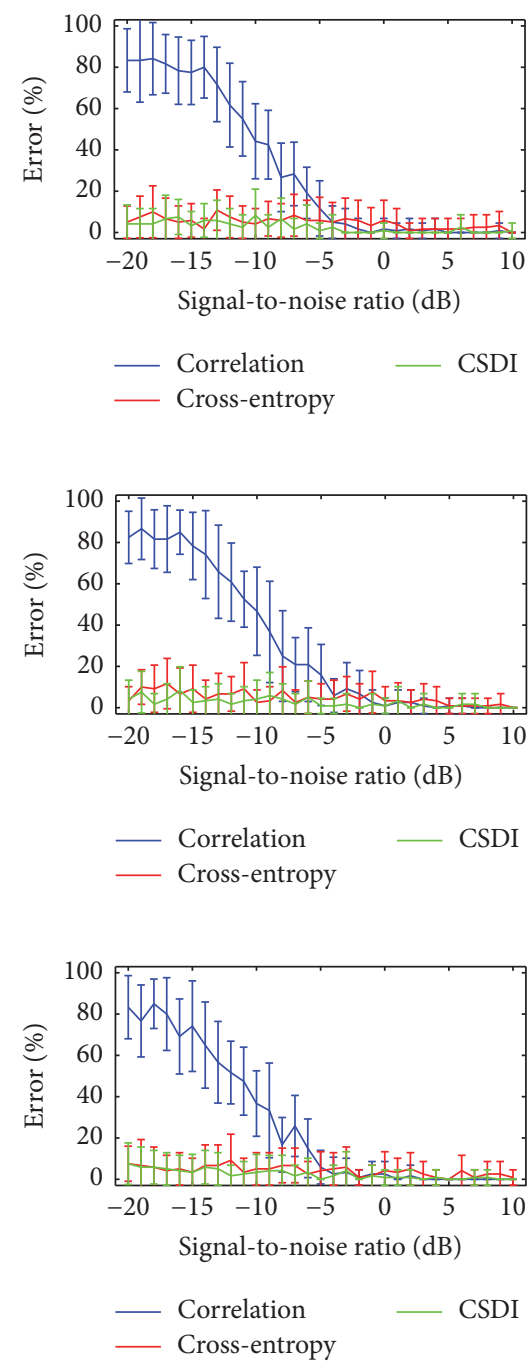

FIGURE 10: Obtained classification error by the pair of filter bank methods and similarity measures with respect to different SNR levels of raw vibration (left), acoustic (middle), and combined vibroacoustic (right) signals from bearing fault test rig experiment.

(above $7 \mathrm{~dB}$ as seen in Figure 10). Yet, these cases of noiseless data are far from being real in usual conditions of machinery operation. The cross-entropy spectral analysis has high accuracy even for very low SNR values for the turbine blade experiment (see Figure 5). However, the best similarity feature is the cumulative spectral density index that achieves the largest performance even for the challenging case of bearing fault setup. Moreover, CSDI outperforms other indexes for SNR values under $-5 \mathrm{~dB}$ regardless of the FBM employed. It is worth noting that CSDI produces the best accuracy with the benefit of a similar computational burden to the other tested indexes for mutual statistical dependence.

(iii) The type of considered machinery faults can be represented by some spectrally localized energies over their extracted narrow subbands, even if the faults behave nonstationary as it is the case for the rolling bearing damage. Consequently, we introduce the use of filter bank methods to carry out the preprocessing stage of acoustic and vibration signals. As a result, all three FBM considered (EMD, WPT, and FFB) allow extracting accurate information in the case of constant shaft speed, providing a comparable computational burden and complexity of implementation.

(iv) Regarding the performed accuracy at low SNR levels, CSDI allows achieving the best performance regardless of the used signal (see Figure 11). Note that the cross-entropy and CSDI measures achieve a comparable accuracy for acoustic signals. In any case, WPT is the best feature set as seen in Table 1 . However, another important aspect is that the vibroacoustic analysis reaches the highest classification rates 


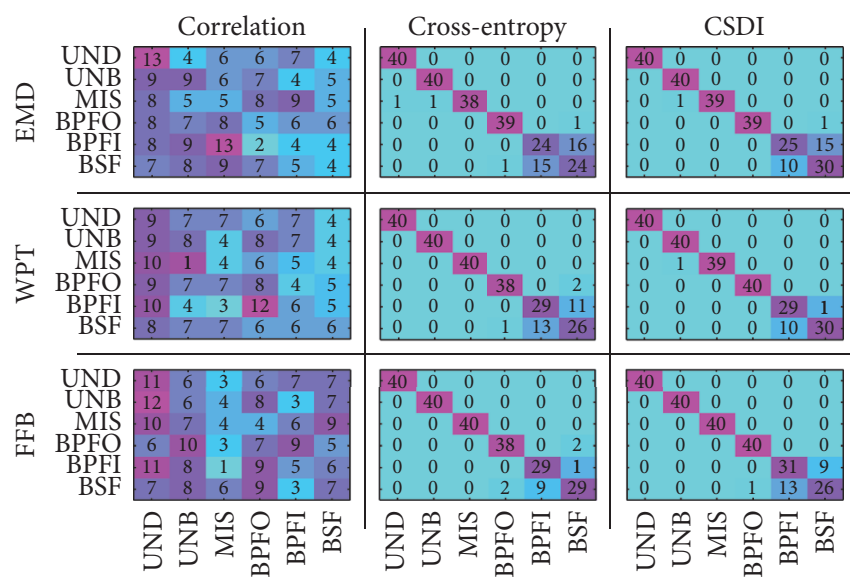

(a) Vibration

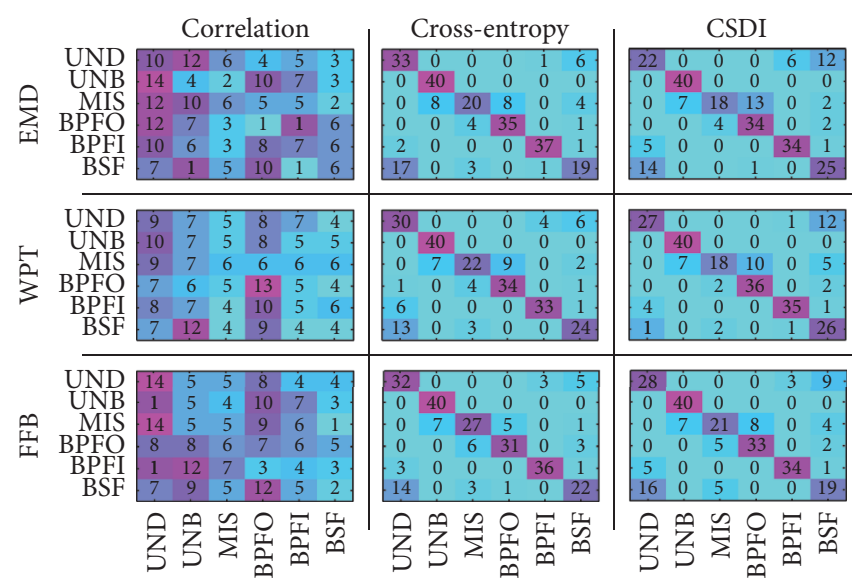

(b) Acoustic

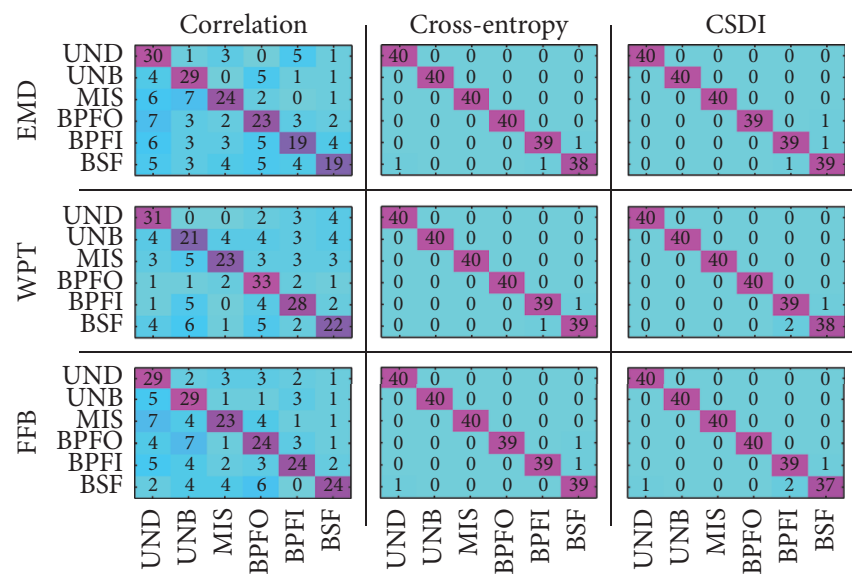

(c) Vibroacoustic

FIGURE 11: Confusion matrix for each pair of filter bank method and similarity measure at $\mathrm{SNR}=-20 \mathrm{~dB}$ using the acquired signals.

using either cross-entropy or CSDI measures (see Figure 10).

\section{Conclusions}

This work introduces a novel methodology to discriminate different failure signatures from rotating machines by measuring the mutual statistical dependence between the input signal and its narrow-band components. Along with the widely used Pearson correlation coefficient and the crossentropy analysis, we propose the cumulative spectral density index that improves the robustness for signals with low SNR. Since the considered machinery faults are spectrally localized over narrow subbands, we carry out enhancement of the acoustic or vibration signals, using EMD, FFB, and WPT algorithms for narrow-band decomposition. Validation over different real-world databases shows that the proposed index of similarity allows improving the performed classifier accuracy for machine fault detection. Besides, the use of vibroacoustic approaches improves the fault detection task in noisy environments, the vibration and acoustic signals being a complementary information for diagnosing multiple rotating machine faults.

As a future work, the authors plan to extend the present analysis to the cases of acquired signals with more complex random structures, for instance, rotating systems under large speed fluctuations.

\section{Competing Interests}

The authors declare that they have no competing interests.

\section{Acknowledgments}

The authors acknowledge the projects "Estudio de la Compatibilidad Electromagnética de los Sistemas de Comunicación. Fase I" developed by Universidad Catolica de Manizales and "Diseño y Desarrollo de un Sistema Prototipo en Línea para el Diagnóstico de Motores de Combustión Interna Diésel en Servicio con Base en Vibraciones Mecánicas. Aplicación a los Sistemas de Transporte Público Masivo" with code "Colciencias 1110-669-46074" and developed by Universidad 
Tecnológica de Pereira and Universidad Nacional de Colombia at Manizales.

\section{References}

[1] N. Baydar and A. Ball, "Detection of gear failures via vibration and acoustic signals using wavelet transform," Mechanical Systems and Signal Processing, vol. 17, no. 4, pp. 787-804, 2003.

[2] D. P. Jena and S. N. Panigrahi, "Automatic gear and bearing fault localization using vibration and acoustic signals," Applied Acoustics, vol. 98, pp. 20-33, 2015.

[3] P. Henriquez, J. B. Alonso, M. A. Ferrer, and C. M. Travieso, "Review of automatic fault diagnosis systems using audio and vibration signals," IEEE Transactions on Systems, Man, and Cybernetics: Systems, vol. 44, no. 5, pp. 642-652, 2014.

[4] M. Amarnath and I. R. Praveen Krishna, "Empirical mode decomposition of acoustic signals for diagnosis of faults in gears and rolling element bearings," IET Science, Measurement \& Technology, vol. 6, no. 4, pp. 279-287, 2012.

[5] J. Lin, "Feature extraction of machine sound using wavelet and its application in fault diagnosis," NDT \& E International, vol. 34, no. 1, pp. 25-30, 2001.

[6] Y. Lei, J. Lin, Z. He, and M. J. Zuo, "A review on empirical mode decomposition in fault diagnosis of rotating machinery," Mechanical Systems and Signal Processing, vol. 35, no. 1-2, pp. 108-126, 2013.

[7] M. Amarnath and I. R. Praveen Krishna, "Local fault detection in helical gears via vibration and acoustic signals using EMD based statistical parameter analysis," Measurement: Journal of the International Measurement Confederation, vol. 58, pp. 154164,2014

[8] R. B. Randall and J. Antoni, "Rolling element bearing diagnostics-a tutorial," Mechanical Systems and Signal Processing, vol. 25, no. 2, pp. 485-520, 2011.

[9] Z. K. Peng, P. W. Tse, and F. L. Chu, "A comparison study of improved Hilbert-Huang transform and wavelet transform: application to fault diagnosis for rolling bearing," Mechanical Systems and Signal Processing, vol. 19, no. 5, pp. 974-988, 2005.

[10] R. Ricci and P. Pennacchi, "Diagnostics of gear faults based on EMD and automatic selection of intrinsic mode functions," Mechanical Systems and Signal Processing, vol. 25, no. 3, pp. 821838, 2011.

[11] J. Obuchowski, A. Wyłomańska, and R. Zimroz, "Selection of informative frequency band in local damage detection in rotating machinery," Mechanical Systems and Signal Processing, vol. 48, no. 1-2, pp. 138-152, 2014.

[12] R. Yan, R. X. Gao, and X. Chen, "Wavelets for fault diagnosis of rotary machines: a review with applications," Signal Processing, vol. 96, pp. 1-15, 2014.

[13] Z. Szabó, "Information theoretical estimators toolbox," Journal of Machine Learning Research, vol. 15, pp. 283-287, 2014.

[14] G. G. Yen and K.-C. Lin, "Wavelet packet feature extraction for vibration monitoring," IEEE Transactions on Industrial Electronics, vol. 47, no. 3, pp. 650-667, 2000.

[15] N. E. Huang, Z. Shen, S. R. Long et al., "The empirical mode decomposition and the Hilbert spectrum for nonlinear and non-stationary time series analysis," Proceedings of the Royal Society of London A: Mathematical, Physical and Engineering Sciences, vol. 454, no. 1971, pp. 903-995, 1998.

[16] J. Ye, "Fault diagnosis of turbine based on fuzzy cross entropy of vague sets," Expert Systems with Applications, vol. 36, no. 4, pp. 8103-8106, 2009.
[17] E. F. Sierra-Alonso, O. Cardona-Morales, C. D. Acosta-Medina, and G. Castellanos-Dominguez, "Spectral correlation measure for selecting intrinsic mode functions," in Progress in Pattern Recognition, Image Analysis, Computer Vision, and Applications, pp. 231-238, Springer, Berlin, Germany, 2014.

[18] G. L. Forbes and R. B. Randall, "Estimation of turbine blade natural frequencies from casing pressure and vibration measurements," Mechanical Systems and Signal Processing, vol. 36, no. 2, pp. 549-561, 2013.

[19] C. Wang, M. Gan, and C. Zhu, "Fault feature extraction of rolling element bearings based on wavelet packet transform and sparse representation theory," Journal of Intelligent Manufacturing, pp. 1-15, 2015.

[20] B. S. Anami, V. B. Pagi, and S. M. Magi, "Wavelet-based acoustic analysis for determining health condition of motorized twowheelers," Applied Acoustics, vol. 72, no. 7, pp. 464-469, 2011.

[21] Y. Lei, Z. He, and Y. Zi, "A new approach to intelligent fault diagnosis of rotating machinery," Expert Systems with Applications, vol. 35, no. 4, pp. 1593-1600, 2008. 


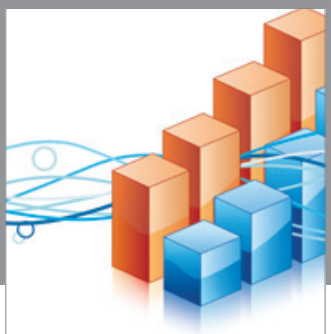

Advances in

Operations Research

vatem alat4

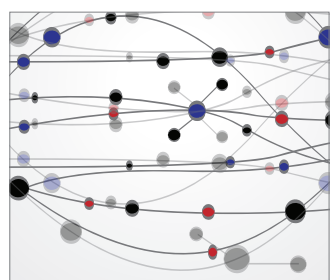

\section{The Scientific} World Journal
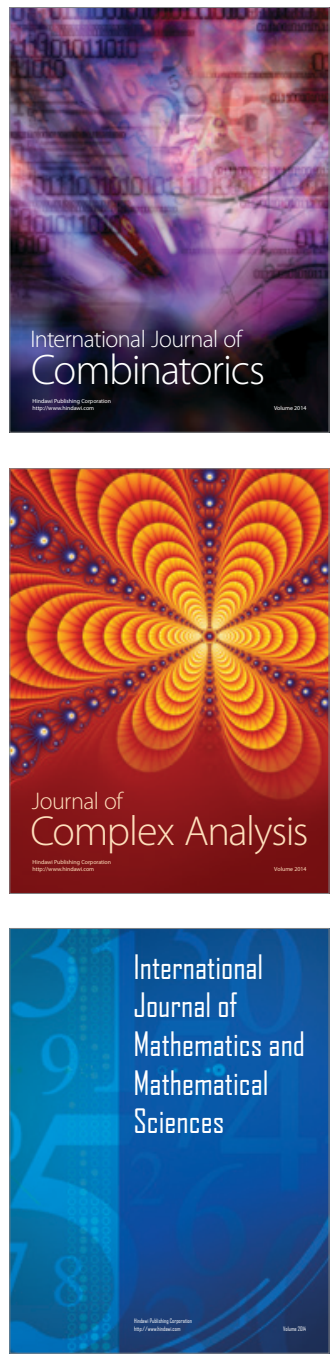
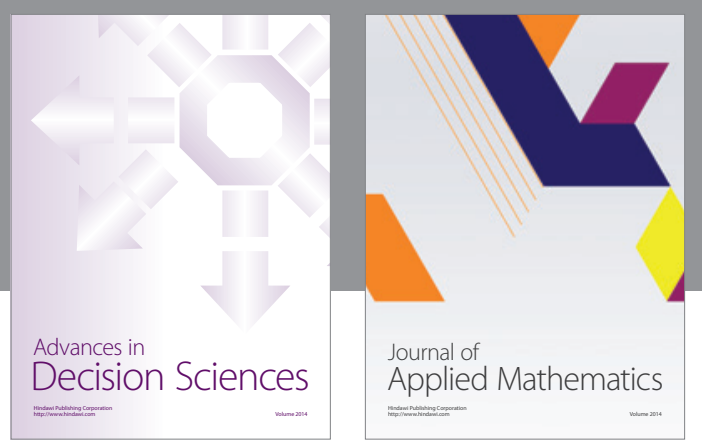

Algebra

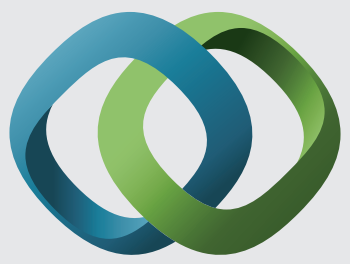

\section{Hindawi}

Submit your manuscripts at

http://www.hindawi.com
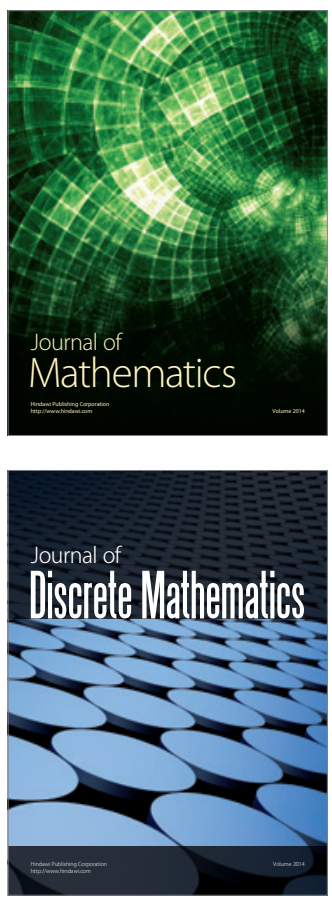

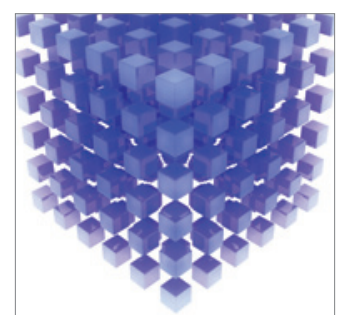

Mathematical Problems in Engineering
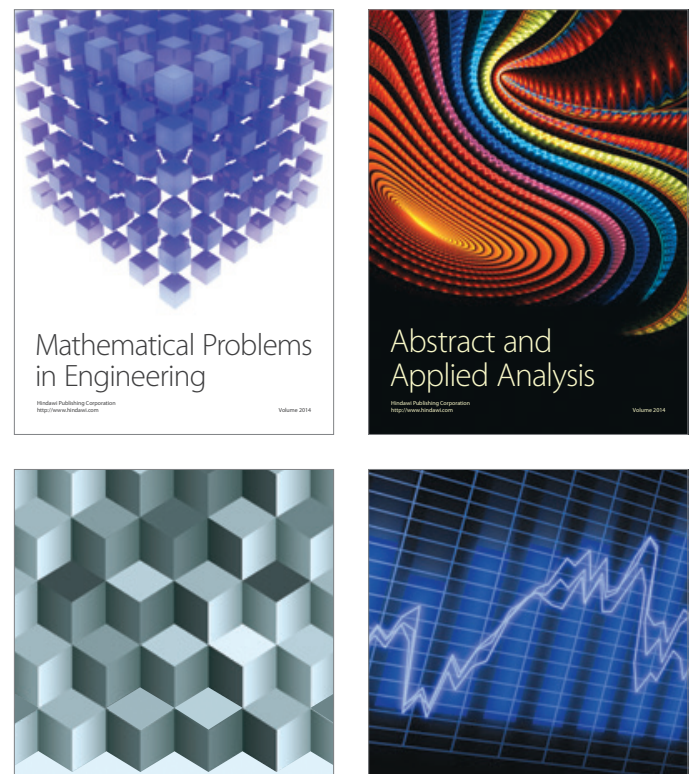

Journal of

Function Spaces

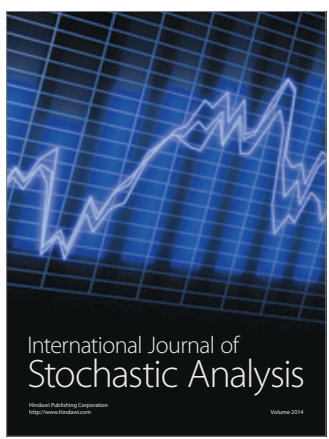

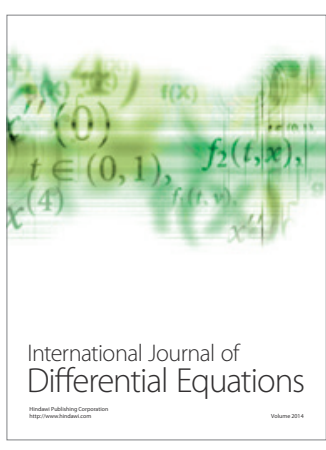
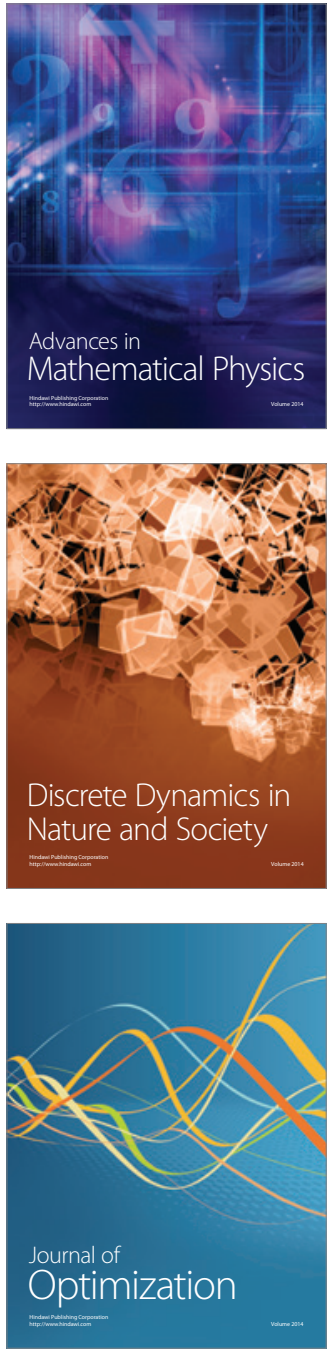